\title{
Time reversal for inclusion detection in one dimensional randomly layered media
}

\author{
D. G. Alfaro Vigo and K. Sølna
}

\begin{abstract}
In this work we discuss detection of changes in a random medium when the measurements are not perfect, i.e. noise from the electronic devices is included. We study a regime in which the typical length scales involved are well-separated. Moreover, since detection procedures based on the analysis of the reflected signals can fail because of the lack of coherency, we introduce a technique based on time reversal which can take advantage of both the coherent time-reversed signal and incoherency associated with the measurement noise.

We use asymptotic analysis of time-reversed signals in a changing medium to guide us in the selection of a statistical decision technique.

The proposed technique is illustrated by a series of numerical simulations. The results show a remarkable agreement with the asymptotic analysis and also highlight the robustness of this technique.
\end{abstract}

\section{Introduction}

Detection and imaging problems arise in various fields of science and technology (e.g. geophysics and medicine). In many situations the detection/imaging procedure relies on the propagation of a probing wave in the medium. In this context, special attention may have to be devoted to the situation when the propagation medium is heterogeneous on a fine scale.

Many of the conventional imaging methods use information provided by the direct reflection from the object of interest. In those cases a coherent reflected signal is produced by a large contrast in for instance the impedance of the background medium, allowing one to detect an object and to estimate the distance from the object to the receiver. Other techniques admit a time reversal or cross correlation interpretation and consequently possess a statistical stabilization property which is important in a heterogeneous environment, see for instance $[\mathbf{3}, \mathbf{4}, \mathbf{5}, \mathbf{7}, \mathbf{9}]$. Here, our focus will be on situations when there is both strong medium heterogeneity as well as strong measurement noise so that the measured signal may not have a any discernible coherent component.

2010 Mathematics Subject Classification. Primary 60G35, 60F05; Secondary 34Q62, 35L05. Key words and phrases. Detection, Time reversal, Random media, Wave propagation.

This work was partially supported by NSF grant 0709389 . 
In [6], a detection/imaging procedure that uses physical time reversal was developed for the case where the macroscopic (effective) equation for the wave energy is a diffusion equation (this occurs for instance in the high-frequency regime when the random fluctuations of the medium are weak and isotropic). Their detection procedure allows for the presence of measurement errors in the time-reversed signal. A high contrast between the diffusion coefficients for the background and the inclusion allows the detection and characterization of the buried object.

In $[\mathbf{8}, \mathbf{1 0}]$ a detection/imaging procedure for a reflector embedded in a randomly layered medium based on physical time reversal was developed. The statistical stability of the time-reversed refocused signal enables this even when the reflected signal is not coherent (i.e. there is no contrast between the impedance of the background and the reflector). The presence of the reflector is detected from the information contained in the time reversal refocusing kernel. In fact, that information is extracted from a continuous family of time reversal refocusing kernels. In $[\mathbf{1}, \mathbf{2}]$ the authors consider the detection problem from the point of view of random matrix theory and with an emphasis on the role measurement noise. In this paper, we are interested in detecting changes in the properties of a randomly layered medium. We analyse a regime in which the probing pulse has a support larger than the random medium fluctuations correlation length, and propagates deep into the medium. We use the measurements obtained after propagating the probing pulse in this medium, during two different periods of time in order to determine whether the properties of the medium has changed or not. When there is no coherent reflection and a relatively strong measurement noise is present the information contained in the measured reflected signals may not be enough to detect the changes. By timereversing the difference of these reflected signals and back-propagating them into the medium we obtain a secondary reflection containing some coherent information. The measurement noise does not have such a focusing to coherent information property and we show how the process thus can enhance the signal to noise ratio. We also show that even in a situation without or with only weak coherent information the procedure can lead to an effective detection scheme.

Under the separation of scales condition considered here, our analysis is restricted to the case where the macroscopic variations of the medium properties are smooth. Consequently, we are only able to detect 'smooth' inclusions with a size several times that of the probing pulse support.

The paper is organized as follows, Section 2 is devoted to the presentation of time reversal in two media, and the generalization of related results obtained in previous works. In particular, we introduce here the time reversal of signal difference procedure that constitutes the basis of our detection technique. In this section we assume that there is no measurement noise. In Section 3, we present the detection technique introducing the appropriate hypothesis test in a context when there is also measurement noise. The results of numerical simulations are presented in Section 4 and show the reliability of the proposed detection technique. The details of the asymptotic analysis justifying the developed technique are presented in the appendixes

\section{Time reversal in random media}

In this section we briefly present the asymptotic description of time reversal in reflection in a changing medium. We introduce and analyze the corresponding 
phenomenon for acoustic waves propagating in a one-dimensional random medium. In this simplified framework, we are able to model and analyze the most important features when a separation of scales condition is satisfied. We believe that these results can be extended to more complex settings. Here we assume that there is no measurement noise, while the situation with measurement noise will be analyzed in Section 3 .

2.1. One-dimensional acoustic model. We consider acoustic wave propagation in a one-dimensional heterogeneous medium modeled by the following equations for the pressure $p$ and the velocity $u$

$$
\begin{aligned}
\varrho(z) \frac{\partial}{\partial t} u(z, t)+\frac{\partial}{\partial z} p(z, t) & =0 \\
\frac{1}{K(z)} \frac{\partial}{\partial t} p(z, t)+\frac{\partial}{\partial z} u(z, t) & =0,
\end{aligned}
$$

where $\varrho$ and $K$ represent density and bulk modulus respectively.

We are interested in the situation where the medium consists of homogeneous and heterogeneous half-spaces separated by a matching interface. Furthermore, we assume that the medium properties in the heterogeneous half-space are random, and there is a separation of scales that allows the identification of large and small scales features. More specifically, the medium properties are characterized by deterministic smoothly varying profiles modeling the large-scale structure (background), about which there are rapidly varying modulating random fluctuations that correspond to the medium micro-structure.

We shall study the situation when an incident pulse can be used to probe the effective (background) medium. More specifically, we consider the asymptotic regime in which

$$
\varepsilon \approx \frac{\text { fluctuations correlation length }}{\text { width of the pulse }} \approx \frac{\text { width of the pulse }}{\text { propagation distance }} \ll 1 \text {. }
$$

This regime is well suited for modeling wave propagation in the earth subsurface, as for instance in exploration geophysics.

By considering an appropriate re-scaling of the space-time variables, and other involved quantities $[\mathbf{7}, \mathbf{1 1}]$, we can assume that the equations above are given in a dimensionless form.

2.2. Time reversal in two media. The time reversal in reflection procedure in two media can be described as follows, a pulse traveling from the right impinges upon the interface $z=0$ of the first medium, the reflected signal is recorded by a time reversal mirror $(\mathrm{TRM})$ during the time interval $\left[0, t_{0}\right]$. Afterwards this reflected signal is time reversed and sent back (i.e. the last recorded part is reemitted first), into the second medium. This procedure generalizes the case where the medium remains unchanged during the whole experiment $[\mathbf{7}, \mathbf{1 3}, \mathbf{1 4}]$.

As stated above, we consider a regime in which the typical wavelength of the incident pulse is longer than the correlation length of the fluctuations, and where the wave propagates over a long distance. In this situation, the long term effect of the medium fluctuations plays an important role in the formation of a coherent refocused signal. Thus, as a result after $t_{0}$ units of time, a coherent pulse traveling to the right emerges at the interface. This phenomenon is known as time reversal refocusing, and the emerging pulse is the so-called refocused pulse. In general, 
its shape is determined by the initial pulse waveform and the media realizations. Nevertheless, there are some interesting situations in which the form of the refocused pulse asymptotically does not depend on the media realizations but only on the media statistics, i.e. it is statistically stable (or self-averaging).

In order to study the asymptotic regime outlined above, we characterize the involved media by the densities $\varrho_{j}(z)$, and bulk moduli $K_{j}(z)$, where the index $j=1,2$ refers to the first or second medium, respectively, as follows

$$
\begin{array}{r}
\varrho_{j}(z)= \begin{cases}\varrho_{j 0}(z)\left(1+\eta_{j}\left(\frac{z}{\varepsilon^{2}}\right)\right), & \text { for } z \leq 0 \\
\varrho_{j 0}(0), & \text { for } z>0\end{cases} \\
K_{j}^{-1}(z)= \begin{cases}K_{j 0}^{-1}(z)\left(1+\mu_{j}\left(\frac{z}{\varepsilon^{2}}\right)\right), & \text { for } z \leq 0 \\
K_{j 0}^{-1}(0), & \text { for } z>0\end{cases}
\end{array}
$$

where $\varrho_{j 0}, K_{j 0}$ and $\eta_{j}, \mu_{j}$ (with $j=1,2$ ), represent the corresponding background properties and random fluctuations, respectively. The unscaled fluctuations $\left(\eta_{j}(\cdot), \mu_{j}(\cdot)\right)$ are mean-zero jointly stationary random processes, that have correlation lengths of order $O(1)$ and rapidly decaying correlation functions. A more complex and realistic model for the random media could be considered by assuming that the random fluctuations also slowly depend on the depth, i.e. they have a multi-scale behavior.

The effective (or background) sound speed and acoustic impedance are given by

$$
c_{j 0}(z)=\sqrt{\frac{K_{j 0}(z)}{\varrho_{j 0}(z)}} \text { and } \zeta_{j 0}(z)=\sqrt{\varrho_{j 0}(z) K_{j 0}(z)},
$$

respectively, with $j=1,2$. We assume in this paper that the effective properties are given by sufficiently smooth functions.

Furthermore, as the incident wave, we assume that the traveling pulse that impinges upon the interface $z=0$ is described as a time signal given by

$$
u_{\text {inc }}(0, t)=-\frac{\zeta_{0}^{-1 / 2}(0) f\left(\frac{t}{\varepsilon}\right)}{2}, \quad p_{\text {inc }}(0, t)=\frac{\zeta_{0}^{1 / 2}(0) f\left(\frac{t}{\varepsilon}\right)}{2}
$$

where $f$ is a smooth function with compact support contained in $[0,+\infty)$. Here the time scaling emphasizes that the typical wavelength of the incident wave is of order $O(\varepsilon)$.

We can describe the time-reversed reflected signal observed in a scaled time window centred at $t_{0}$ as

$$
u_{\mathrm{ref}}^{\mathrm{TR}}\left(0, t_{0}+\varepsilon s\right)=\frac{\zeta_{0}^{-1 / 2}(0) B_{t_{0}}^{\varepsilon, \mathrm{TR}}(s)}{2}, \quad p_{\mathrm{ref}}^{\mathrm{TR}}\left(0, t_{0}+\varepsilon s\right)=\frac{\zeta_{0}^{1 / 2}(0) B_{t_{0}}^{\varepsilon, \mathrm{TR}}(s)}{2}
$$

where $B_{t_{0}}^{\varepsilon, \mathrm{TR}}(\cdot)$ is a random function that depends on the media properties through the reflection coefficients of the two media.

Important information on the time-reversed acoustic field for $\varepsilon$ small can be obtained by an asymptotic analysis of the random signal $B_{t_{0}}^{\varepsilon, \mathrm{TR}}(\cdot)$ as $\varepsilon \rightarrow 0$.

2.3. Time reversal asymptotics and statistical stability. We continue here the discussion of the time time reversal in two media configuration introduced in the previous section. We briefly present the main results of the asymptotic analysis of the problem and highlight a simplified situation which is important 
for our application, while a more general and detailed analysis is presented in the appendix.

Under some technical assumptions regarding the random fluctuations, we have that $B_{t_{0}}^{\varepsilon, \mathrm{TR}}(\cdot)$ converges in distribution as $\varepsilon \downarrow 0$ to the random signal

$$
B_{t_{0}}^{\mathrm{TR}}(s)=\frac{1}{2 \pi} \int \mathrm{e}^{\mathrm{i} \omega s} \widehat{K_{12, t_{0}}^{\mathrm{TR}}}(\omega) \bar{f}(\omega) \mathrm{d} \omega .
$$

where the (random) time reversal refocusing kernel $K_{12, t_{0}}^{\mathrm{TR}}$ is related to the asymptotics of a the solution of a backward Itô stochastic partial differential equation, see appendix A.

As a model for simple inclusions we consider the situation where the smooth function $\delta c(z)=c_{20}(z)-c_{10}(z)$ for $z \leq 0$, does not change sign (i.e. $\delta c(z) \geq 0$ or $\leq 0$ for $z \leq 0$ ) and is compactly supported. Moreover, if we additionally assume that the sum of the random fluctuations for the density and the bulk modulus are fully correlated for the two media, then we obtain that the refocused signal is statistically stable (see the appendix for details). This condition could be weakened by considering that the random fluctuations have a multi-scale behavior, and their referred sums are fully correlated in the region outside the support of $\delta c(\cdot)$ (i.e. where the medium does not change). Physically, this stability can be understood as the inclusion making the response of the modified part of the random medium incoherent relative to that associated with the non-deformed medium so that it contributes to the refocused signal at a lower order.

We remark that in the statistically stable scenario, we have the convergence in probability of the time-reversed signal, whereas in the general situation the convergence occurs in distribution. This means that in the former case the refocused signal $B_{t_{0}}^{\varepsilon, \mathrm{TR}}(\cdot)$ (for a small $\varepsilon$ ) remains close to the limiting deterministic signal $B_{t_{0}}^{\mathrm{TR}}(\cdot)$.

2.4. Time reversal of the signal difference. Next, we introduce a slightly different configuration which is the one that we will use for the detection. We introduce the time reversal of the signal difference corresponding to the two media. The reflections of similar, ideally the same, pulses that impinge upon the interfaces of the initial and modified media are first recorded. The difference of these reflected signals is time reversed and sent back into the modified medium by using a TRM. The corresponding secondary reflections that emerge at the interface are called time-reversed difference reflection. The resulting signal correspond to the difference of two time-reversed signals, the first one obtained by time reversal in the modified medium (that remains unchanged during the procedure) and the second one corresponding to time reversal in a changing medium (i.e. involving these two media).

The time-reversed difference reflection signal $B_{t_{0}}^{\varepsilon, T R D}(\cdot)$ can be characterized in a similar way as before, and we get that it converges in distribution as $\varepsilon \downarrow 0$ to the random signal

$$
B_{t_{0}}^{\mathrm{TRD}}(s)=\frac{1}{2 \pi} \int \mathrm{e}^{\mathrm{i} \omega s} \widehat{K_{12, t_{0}}^{\mathrm{TRD}}}(\omega) \bar{f}(\omega) \mathrm{d} \omega
$$

where

$$
K_{12, t_{0}}^{\mathrm{TRD}}(\omega)=K_{22, t_{0}}^{\mathrm{TR}}(\omega)-K_{12, t_{0}}^{\mathrm{TR}}(\omega),
$$


the subindices indicate that the kernels correspond to standard time reversal in the second medium and time reversal in two media respectively.

\section{Time reversal detection}

3.1. Detection problem. We now focus on the problem of detecting inclusions in a highly heterogeneous medium. First of all, we remark that by inclusion we understand changes in the effective properties of the medium. Furthermore, we assume that the inclusions satisfy the following properties. The size of the inclusions should be several times larger than the probing pulse width, and smoothly varying on this scale. The inclusion increases (or decreases) the effective sound speed of the medium.

We shall probe the medium during different periods of time in order to know if any change has occurred in the medium properties. More specifically, if we think of the medium during these two periods of time, as been modeled by equations (2.2) (for media ' 1 ' and ' 2 '), we are interested in determining if the effective sound speeds of the two media are different, i.e. $c_{20}(z) \neq c_{10}(z)$.

To probe the media one uses an incident pulse that scales as (2.4), and search for information in the reflected signal but when there is no coherent reflection, all the information is hidden in the scattered signals produced by the random fluctuations of the medium, and a straightforward application of a detection technique is difficult to use, because of the low signal to noise ratio. Nevertheless, the statistics of the reflected signals are well understood and it is possible to extract information about the medium properties (see $[7,12,17,18]$ ).

We introduce here a method based on the time reversal difference procedure presented in section 2.4 and a hypothesis testing technique, considering the situation where measurement noise is present in the data. Our method is advantageous relative to just using the reflected signals. Since the time reversal difference procedure yields a coherent signal, one usually has a high signal to noise ratio and therefore standard detection techniques perform well. Moreover, when the refocused signal is statistically stable the influence of the random medium fluctuations are controlled and consequently good performance of the detection technique is expected. We shall show that our approach works well in very noisy environments.

3.2. Measured time-reversed difference reflection and its asymptotics. As a result of the data acquisition process, some errors are introduced in the measured quantities. The quantities we are interested in are signals smoothly varying on the scale $\varepsilon$. We model the error introduced during a direct measurement of a time signal as an additive 'noise' varying on the scale $\varepsilon^{a}$ with $a>0$, that is the measured signal $g_{\text {meas }}^{\varepsilon}(t)$ associated with the actual signal $g^{\varepsilon}(t)$ is given as

$$
g_{\text {meas }}^{\varepsilon}(t)=g^{\varepsilon}(t)+\nu\left(\frac{t}{\varepsilon^{a}}\right)
$$

where $\nu(\cdot)$ is a mean zero, stationary Gaussian random process. Note that when $a=1$, the noise fluctuates on the same scale as the incoherent wave reflections. Furthermore, we consider that this process has an integrable autocorrelation function which has the representation

$$
E\{\nu(s) \nu(0)\}=\int \mathrm{e}^{\mathrm{i} \omega s} F_{\nu}(\omega) \mathrm{d} \omega
$$


where $F_{\nu}(\cdot) \geq 0$ is the power spectral density [19], and $E\{\cdot\}$ represents expectation. Furthermore, the measurement error intensity is characterized by

$$
\sigma_{\nu}^{2}=E\left\{\nu^{2}(0)\right\}=\int F_{\nu}(\omega) \mathrm{d} \omega .
$$

Assuming that the direct measurement errors introduced during the time reversal procedure are statistically independent, we get that

$$
B_{t_{0}, \text { meas }}^{\varepsilon, \text { TRD }}(s)=B_{t_{0}}^{\varepsilon, \text { TRD }}(s)+B_{t_{0}, \nu}^{\varepsilon}(s)
$$

The first term in this decomposition represents the actual time reversal signal difference (when no measurement errors are introduced during the process) and the second is associated with measurements errors. It arises from the propagation of the difference of the direct measurements noise associated with the primary reflections and the error in the direct measurement of the time-reversed difference reflection.

When $a \neq 1$, in the asymptotic limit $\varepsilon \downarrow 0$, the term associated with the measurement errors can be filtered out (see appendix for details). Thus, we shall focus on the case where $a=1$. Using the properties of the involved random processes, one can get that $B_{t_{0}, \nu}^{\varepsilon}(s)$ converges in distribution as $\varepsilon \downarrow 0$ to a stationary, Gaussian random process with mean $B_{t_{0}}^{\mathrm{TRD}}(s)$ given by (2.6) and covariance function

$$
C_{t_{0}, \nu}(s)=\int \mathrm{e}^{\mathrm{i} \omega s}\left(1+2 \widehat{K_{2, t_{0}}^{\mathrm{R}}}(\omega)\right) F_{\nu}(\omega) \mathrm{d} \omega,
$$

where $K_{2, t_{0}}^{\mathrm{R}}(\cdot)$ is related to the asymptotics of a backward Kolmogorov equation whose coefficients are associated with the statistics of the second (changed) random medium, see appendix B.

\subsection{Statistical test.}

3.3.1. Hypothesis testing formulation. The detection problem can be stated as a hypothesis testing problem for the following general hypotheses:

$\mathrm{H}_{0}$ : there are no changes in the medium (null hypothesis)

$\mathrm{H}_{\mathrm{a}}$ : the medium has changed (alternate hypothesis)

According to the general theory of hypothesis testing [21], the statistical test consists of a procedure to decide whether the null hypothesis can be accepted or rejected. In general, a region in the space where the sample lives is selected and when the sample belongs to it the hypothesis is rejected. This region is the so-called rejection region. In a test two types of errors can be made. Type I errors correspond to rejecting $\mathrm{H}_{0}$ when it is correct (false alarm) and type II errors to accepting $\mathrm{H}_{0}$ when it is false (missed detection). Their probabilities play an important role in the design of a test.

The probability of type I errors is given by

$$
\alpha=\operatorname{Pr}\left\{\text { rejecting } \mathrm{H}_{0} \mid \mathrm{H}_{0} \text { is true }\right\} \text {, }
$$

while the probability of type II errors is expressed as

$$
\beta=\operatorname{Pr}\left\{\text { accepting } \mathrm{H}_{0} \mid \mathrm{H}_{\mathrm{a}} \text { is true }\right\} .
$$

Since generally, both errors can not be kept small at the same time a guideline for designing the test is to select the rejection region in such a way that the probability of type II errors $(\beta)$ is minimized when the probability of type I errors $(\alpha)$ is fixed. The probability $\alpha$ is called the level of significance of the test. The success of the test (probability of detection) is called the power of the test and equals $1-\beta$. In 
detection applications it is usually presented graphically as the Receiver Operating Curve (ROC) that represents the power of the test as a function of the level of significance.

The asymptotic description of the measured time reversal difference signal as a Gaussian random process presented above allows us to select an appropriate statistical test for this detection problem. In what follows, we consider the detection problem for the asymptotic characterization of the measured time reversal difference signal.

We consider the (finite) discrete time sampling of the time reversed signal $\mathbf{x}=\left(B_{t_{0}, \text { meas }}^{\text {TRD }}\left(s_{1}\right), \cdots, B_{t_{0}, \text { meas }}^{\text {TRD }}\left(s_{M}\right)\right)^{t}$ with uniform sampling rate $h=s_{j+1}-s_{j}$ and centred at $s=0$ (i.e. $\left(s_{1}+s_{M}\right) / 2=0$ ). The hypotheses can be reformulated as follows

$\mathrm{H}_{0}: \mathbf{x}$ is a sample of the random variable $\mathbf{X}_{0} \sim \mathcal{N}\left(\mathbf{0}, \mathbf{C}_{0}\right)$

$\mathrm{H}_{\mathrm{a}}: \mathbf{x}$ is a sample of a random variable $\mathbf{X}_{a} \sim \mathcal{N}\left(\boldsymbol{\mu}_{\mathrm{a}}, \mathbf{C}_{\mathrm{a}}\right)$,

where the mean vector $\boldsymbol{\mu}_{\mathrm{a}}=\left(B_{t_{0}}^{\mathrm{TRD}}\left(s_{1}\right), \cdots, B_{t_{0}}^{\mathrm{TRD}}\left(s_{M}\right)\right)^{t}$ with the $B_{t_{0}}^{\mathrm{TRD}}(\cdot)$ given by (2.6) and the elements of the covariance matrices $\left(\mathbf{C}_{0}\right)_{i j},\left(\mathbf{C}_{\mathrm{a}}\right)_{i j}$ are of the form $C_{t_{0}, \nu}((j-i) h)$ given by (B.3) with the kernel $K_{t_{0}}^{\mathrm{R}}(\cdot)$ corresponding to the initial and second (changed) media, respectively.

In general, the covariance matrix $\mathbf{C}_{0}$ is unknown, however it can be estimated by performing time reversal experiments in the unchanged medium. (In the case of a homogeneous medium it can be explicitly computed from equation (B.3).) Thus, we now assume that $\mathbf{C}_{0}$ is given. Concerning the covariance matrix $\mathbf{C}_{\mathrm{a}}$, we assume that a set of admissible matrices $\mathcal{S}_{M}$ is given (see the appendix for details).

Therefore, we can reformulate the problem as follows: given a sample $\mathbf{x}$ of a random variable distributed as $\mathcal{N}(\boldsymbol{\mu}, \mathbf{C})$ test the hypotheses $\mathrm{H}_{0}$ vs. $\mathrm{H}_{\mathrm{a}}$, where

- $\mathrm{H}_{0}: \boldsymbol{\mu}=\mathbf{0}$ and $\mathbf{C}=\mathbf{C}_{0}$

- $\mathrm{H}_{\mathrm{a}}: \boldsymbol{\mu} \neq \mathbf{0}$ and $\mathbf{C} \in \mathcal{S}_{M}$.

3.3.2. A two-sided likelihood ratio test. As starting point for selecting the rejection region we use a likelihood ratio $(L R)$ test $[\mathbf{2 1}]$ based on the statistic $Q_{M}(\mathbf{x})=$ $\mathbf{x}^{t} \mathbf{C}_{0}^{-1} \mathbf{x}$ that has a $\chi^{2}$-distribution with $M$ degrees of freedom under the hypothesis $H_{0}$. However, the analysis of this test and its asymptotics as $M \rightarrow \infty$, reveals that it is biased, see appendix B. To avoid this situation, we propose a two-sided LR test whose rejection region at significance level $\alpha$ is given by

$$
R_{\alpha}=\left\{\mathbf{x}: Q_{M}(\mathbf{x}) \leq \chi_{M}^{2}(\alpha / 2) \text { or } Q_{M}(\mathbf{x}) \geq \chi_{M}^{2}(1-\alpha / 2)\right\},
$$

where $\chi_{M}^{2}(\cdot)$ represents the inverse of the cumulative $\chi^{2}$-distribution function with $M$ degrees of freedom. Furthermore, an asymptotic analysis leads to the conclusion that for a fixed value of the significance level $\alpha>0$ and under suitable conditions, the power of the test

$$
P(\alpha ; \boldsymbol{\mu}, \mathbf{C})=\operatorname{Pr}\left\{\mathbf{x} \in R_{\alpha} \mid \mathbf{x} \sim \mathcal{N}(\boldsymbol{\mu}, \mathbf{C})\right\} \rightarrow 1
$$

as $M \rightarrow \infty$. Moreover, asymptotically the rate of convergence does not depend on the measurement noise intensity nor the time-reversed signal energy. This means that by using a sufficiently large sample we can achieve the required performance of the detection procedure.

3.3.3. Sensitivity on the inclusion characteristics. Next, we analyze how the characteristics of the inclusion affects the quality of the detection. More exactly, we estimate how the number of sample points necessary to achieve a successful 
detection depends on the inclusion properties. This analysis is based on the combination of some heuristic arguments and simple asymptotic results, see appendix B.

Let us consider an inclusion that changes the sound speed of the background medium from $c_{1}$ to $c_{2}$, and has size $\Delta z \leq l=c_{1} t_{0} / 2$. Furthermore, consider that the number

$$
\Xi=\frac{\Delta z}{l} \frac{\left|c_{2}-c_{1}\right|}{c_{2}} \ll 1
$$

Note that this condition is fulfilled, for instance, when the relative variation of the sound speed and the relative size of the inclusion are small.

Under this circumstance, for a level of significance $\alpha>0$ we expect a probability of detection better than $1-\tilde{\beta}$ when the number of sample points satisfies

$$
M \gtrsim 2\left(\frac{\Phi^{-1}(1-\alpha / 2)+\Phi^{-1}(1-\tilde{\beta})}{\Xi}\right)^{2},
$$

and the sample rate $h$ is sufficiently small.

This gives a rough estimate of the size of the sample. In the numerical examples of the next section we used less than half the estimated number of sampling points, nevertheless we achieved an excellent rate of success.

\section{Numerical results}

In order to establish how well the introduced detection technique works we carry out several Monte-Carlo simulations by numerically solving the model equations (2.1). In doing this we address several key aspects of our approach to the detection problem. First, we illustrate the reliability of this detection technique by showing that the probability of detection observed in the simulations, is in complete agreement with the results predicted by the asymptotic theory, despite the fact that in simulations the small parameter $\varepsilon$ is finite. Finally, we show the robustness of this technique to assumptions made in the asymptotic analysis.

4.1. Detecting an inclusion. In this series of simulations we address the reliability of the proposed detection technique. We illustrate how using time reversal enhances the signal to noise ratio when compared with using only the reflected signals. Furthermore, we establish that the level of success of this detection test predicted from the asymptotic theory is actually achieved in the numerical simulations.

We consider the detection of an inclusion, that extends from $z=-25$ to $z=$ -50 , on an initial medium with a homogeneous background with $\varrho_{10}=K_{10}=$ 1. The relative changes induced by the inclusion in the background and local sound speed are approximately of $12.3 \%$ and $12.9 \%$, respectively. We only consider random fluctuations of the media density which have a $30 \%$ maximum intensity and a $17 \%$ standard deviation. One realization of the profiles of the sound speed before and after inclusion is presented in figure 1. In the time reversal numerical procedure the incident pulse is a Gaussian of amplitude and width equal to one unit, and the recording time is $t_{0}=90$ time units. In the scaling we have chosen the small parameter $\varepsilon \approx 0.1$. The numerical solution of the corresponding acoustic equations is carried out using a Lagrangian numerical scheme with discretization stepsizes $\Delta t=\Delta z=0.01$ (see details in [25]). 
First, we carry out several time reversal experiments corresponding to an inclusion as depicted in figure 1 (upper left corner plot) for different levels of the measurement noise $\left(\sigma_{\nu}=0.05-0.5\right)$. In figure 2 we plot the signal to noise ratio associated with the reflected signals and the time-reversed difference reflection, respectively, with respect to the measurement noise intensity. It is apparent from the figure that time reversal enhances the signal to noise ratio, emphasizing the advantage of this approach.
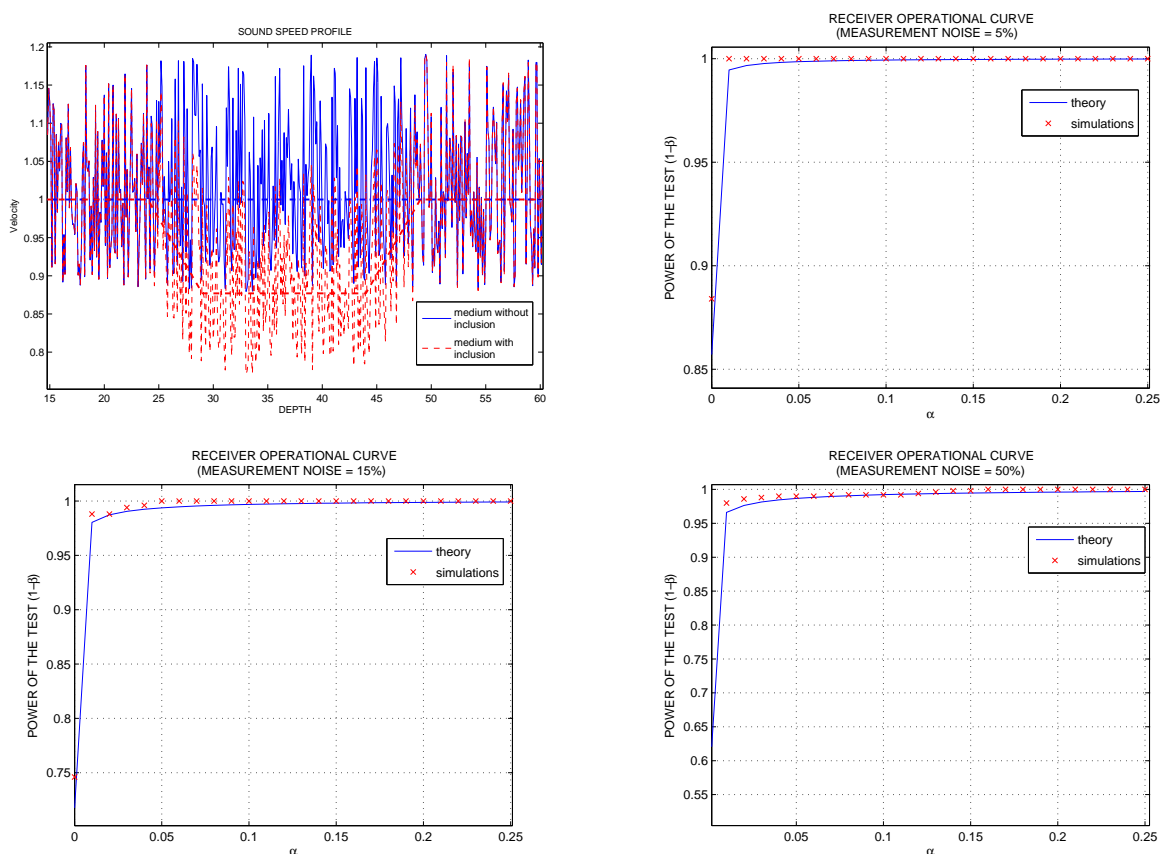

FiguRE 1. One realization of the profile of the propagation velocity corresponding to an inclusion. Theoretical Receiver Operating Curves (ROCs) and probability of detection obtained from a series of Monte-Carlo simulation with 500 realizations of the time reversal experiment using different values of the measurement noise $\left(\sigma_{\nu}=0.05,0.15\right.$ and 0.50$)$.

We made three sets of Monte-Carlo simulations corresponding to three different levels of the measurement noise $\left(\sigma_{\nu}=0.05,0.15\right.$ and 0.50$)$ with 500 realizations of the time reversal experiment per set. In figure 1 we compare the ROCs corresponding to the asymptotic theory and the simulations. The theoretical ROCs are obtained from the appropriate expression of the power of the test $P(\cdot)$ by numerically evaluating the integral (B.10) after estimating the required parameters $\boldsymbol{\xi}$ and $\boldsymbol{\lambda}$ from the corresponding Monte-Carlo simulations. The ROC corresponding to simulations is obtained by computing the rate of success in each set of Monte-Carlo simulations for different levels of significance $(0.001 \leq \alpha \leq 0.25)$. Moreover, the rate of rejection obtained in the simulations is in complete agreement with the corresponding level of significance. The test statistics are computed from samples with 


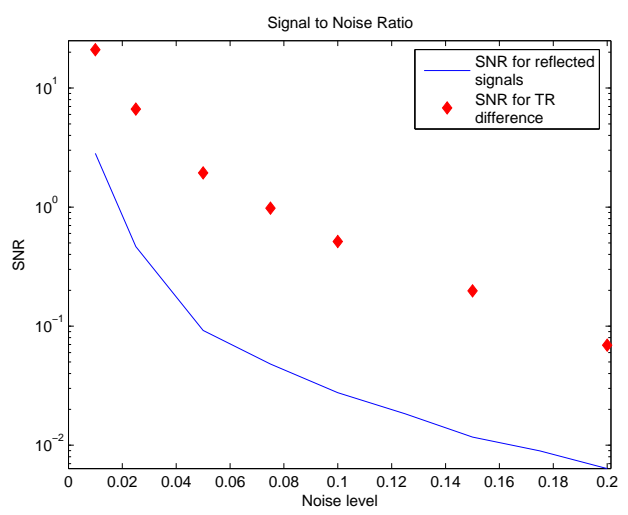

FiguRE 2. Signal to noise ratio corresponding to the reflected signals and the time-reversed difference reflection.

size $M=200$ and time sampling rate $h=0.01$. From this figure we conclude that there is a remarkable agreement between the ROCs from the asymptotic theory and the simulations. It is also apparent that the probability of detection is not very sensitive to the intensity of the measurement noise as predicted by our theory.

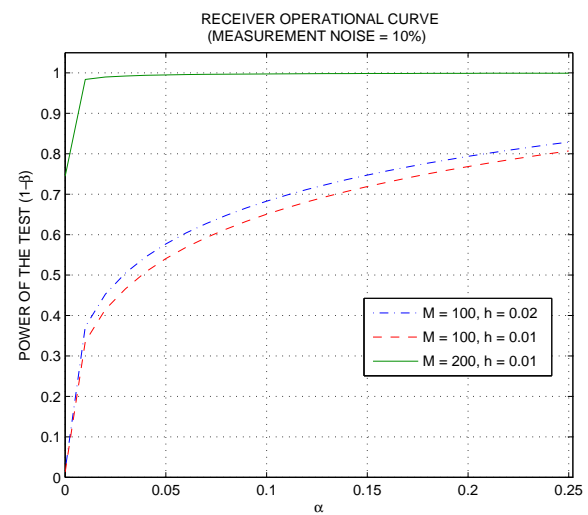

Figure 3. Comparison of the Theoretical Receiver Operating Curves (ROCs) for different sampling rates $(h=0.01$ and 0.02$)$ and different sample size $(M=100$ and 200).

Finally, we briefly illustrate the influence of the time sampling rate $h$ and the sample size $M$. In figure 3 , the three ROCs corresponding to $(M, h)=(100,0.02)$, $(100,0.01)$ and $(200,0.01)$, respectively, are shown. We can see that doubling the sample size produced a remarkable increase of the power of the test, whereas halving the sampling rate slightly reduced the power of the test.

4.2. Detecting a fluctuating slab. The next example concerns the robustness of the proposed detection technique. Recall that the corresponding statistical test was obtained under the assumption that the time-reversed difference reflection 
is statistically stable. However, this happens under very specific conditions, for instance when one has an increasing/decreasing velocity perturbations. Furthermore, in typical situations we do not know that these conditions are fulfilled. Nonetheless, we show that the proposed detection technique is reliable under less restrictive conditions, namely in the case where the change occurs only in the fluctuations, a situation which still can be captured by our hypothesis based formulation.

We let the fluctuations change only in the finite slab from $z=-24$ to $z=-44$ of the medium, while the background propagation velocity remains unchanged and equal to 1.

The first plot in figure 4 represents one realization of the profile of the sound speed. The time reversal setup is similar to the one in the previous section, we use the same incident Gaussian pulse, recording time $t_{0}=90$ and a small parameter $\varepsilon \approx 0.1$.
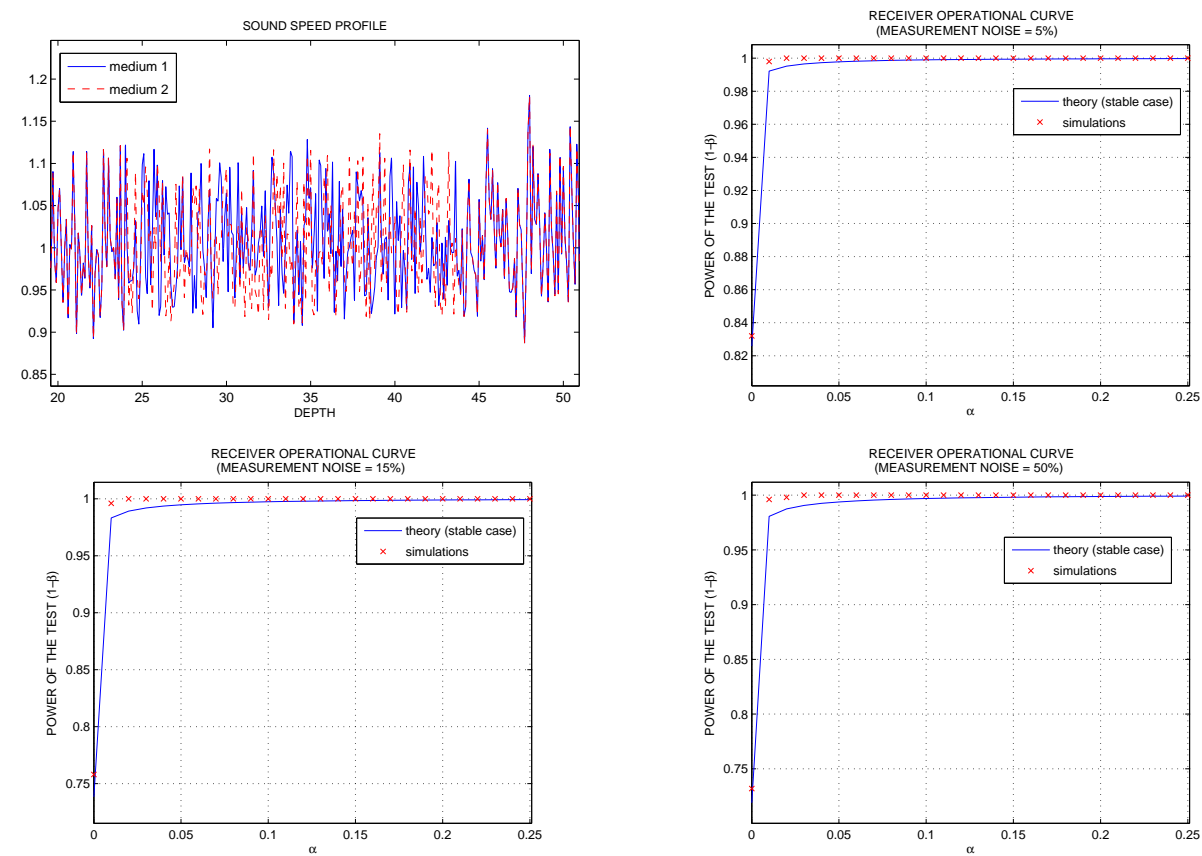

Figure 4. One realization of the profile of the propagation velocities corresponding to two media that only differ in terms of the fluctuations. Theoretical ROCs and probability of detection obtained from a series of Monte-Carlo simulation with 500 realizations of the time reversal experiment using different values of the measurement noise $\left(\sigma_{\nu}=0.05,0.15\right.$ and 0.50$)$.

We run three sets of Monte-Carlo simulations corresponding to the measurement noise levels $\sigma_{\nu}=0.05,0.15$ and 0.50 , with 500 realizations each, to estimate all the necessary parameters in order to apply the statistical test and obtain its probability of detection for different values of the level of significance $(0.001 \leq \alpha \leq 0.25)$. The estimated parameters are also used to get the curves of the power $P(\cdot)$ using the equations corresponding to the statistically stable case. 
The results are presented in figure 4. There is a remarkable agreement between the (statistically stable) power of the test curve and the probability of detection obtained in the Monte-Carlo simulations. The results are slightly better than those presented in the previous section, demonstrating that this approach may be very efficient for estimation in certain scaling regimes. Moreover, as in the previous section the results are not very sensitive to the intensity of the measurement noise.

\section{Concluding remarks}

In this paper, we introduce a statistical technique for the detection of inclusions in a random medium, that takes into effect measurement errors. This detection technique relies on a time-reversal procedure and a statistical hypothesis testing approach. For the derivation of the statistical test, we take advantage of the asymptotic behaviour of the time-reversed difference signal as a small parameter $\varepsilon$, approaches zero.

The statistical test was specifically designed for the case where the time-reversed difference reflection satisfies the celebrated statistical stabilization property. We established this property for a situation that models a general class of inclusions.

Through a series of Monte-Carlo simulations we established the reliability of this detection technique when $\varepsilon$ is small but finite, and we also established its robustness concerning the statistical stability property. More specifically, we showed that the probability of success of this detection test observed during simulations are in a remarkable agreement with those predicted by the asymptotic theory. Moreover, similar results are obtained in simulations where the statistical stability property is no longer valid. We also showed that by increasing the size of the sample we improve the performance of the detection technique.

\section{Appendix A. Asymptotic analysis of time reversal in a changing medium}

This appendix contains a generalization of some results that were presented in $[\mathbf{1 3}, \mathbf{1 4}]$, concerning time reversal in reflection in a changing medium. More specifically, we consider the case where the background as well as the random fluctuations of the media involved in the time reversal procedure are different, but assume that the homogeneous half-space remains unchanged. In the cited references, the background properties also remain unchanged. In this appendix we assume no measurement noise, while we analyze the situation with measurement noise and the detection test in appendix B.

Let the involved media be characterized by the densities and bulk moduli described by (2.2), and their corresponding effective (or background) sound speed and acoustic impedance given by (2.3). We recall that the scattering effect of each inhomogeneous half-space is characterized in frequency space, by the medium reflection coefficients $R_{j}^{\varepsilon}(\omega), j=1,2$ that solve their corresponding random Riccatti equations $[\mathbf{7}, \mathbf{1 3}]$.

We let the impinging pulse be given by (2.4) and assume that the TRM is characterized by a cutoff function $G_{t_{0}}(\cdot)$ supported on the recording time interval $\left[0, t_{0}\right]$ (or rapidly decaying outside it). 
Observing the time-reversed reflected acoustic field at the interface $\left(u_{\mathrm{ref}}^{\mathrm{TR}}, p_{\mathrm{ref}}^{\mathrm{TR}}\right)$, in a scaled time window centered at $t_{0}$, we get that

$$
u_{\mathrm{ref}}^{\mathrm{TR}}\left(0, t_{0}+\varepsilon s\right)=\frac{\zeta_{0}^{-1 / 2}(0) B_{t_{0}}^{\varepsilon, \mathrm{TR}}(s)}{2}, \quad p_{\mathrm{ref}}^{\mathrm{TR}}\left(0, t_{0}+\varepsilon s\right)=\frac{\zeta_{0}^{1 / 2}(0) B_{t_{0}}^{\varepsilon, \mathrm{TR}}(s)}{2},
$$

where

$$
B_{t_{0}}^{\varepsilon, \mathrm{TR}}(s)=\frac{1}{(2 \pi)^{2}} \iint \mathrm{e}^{\mathrm{i}\left(\omega-\varepsilon \frac{h}{2}\right) s} \overline{\hat{f}}\left(\omega+\frac{\varepsilon}{2} h\right) \overline{\hat{G}_{t_{0}}}(h) R_{1}^{\varepsilon}\left(\omega+\frac{\varepsilon}{2} h\right) \overline{R_{2}^{\varepsilon}}\left(\omega-\frac{\varepsilon}{2} h\right) \mathrm{d} \omega \mathrm{d} h .
$$

Notice that the signal $B_{t_{0}}^{\varepsilon, \mathrm{TR}}(\cdot)$ is random and depends on the properties of the two media through the interface reflection coefficients.

A.1. Characterization of the limiting refocused pulse. A characterization of the refocused pulse for $\varepsilon \ll 1$, is obtained by an asymptotic analysis (as $\varepsilon \downarrow 0$ ) of the time-reversed reflected signal $B_{t_{0}}^{\varepsilon, \mathrm{TR}}(s)$. This analysis relies on the characterization of the limiting statistical moments of this signal using a diffusionapproximation theorem and Itô formula for stochastic differential equations. The calculations are analogous to those presented in $[\mathbf{1 3}]$.

We have that the limit in distribution of the random signal $B_{t_{0}}^{\varepsilon, \mathrm{TR}}(s)$ as $\varepsilon \downarrow 0$ is given by the random signal $B_{t_{0}}^{\mathrm{TR}}(s)$

$$
B_{t_{0}}^{\mathrm{TR}}(s)=\left(K_{12, t_{0}}^{\mathrm{TR}}(\cdot) * f(-\cdot)\right)(s)=\frac{1}{2 \pi} \int \mathrm{e}^{\mathrm{i} \omega s} \widehat{K_{12, t_{0}}^{\mathrm{TR}}}(\omega) \overline{\hat{f}}(\omega) \mathrm{d} \omega
$$

where $*$ represents convolution in time, and the (random) time reversal refocusing kernel $K_{12, t_{0}}^{\mathrm{TR}}(\cdot)$ is defined by equation (A.7) whose detailed description follows from the ensuing analysis. It is associated with the asymptotic behaviour of the solution of an Itô partial differential equation, a detailed description is presented below.

Let us define the differential operator

$$
\begin{array}{r}
\mathcal{L}_{z}=h\left(\frac{1}{c_{10}(z)}+\frac{1}{c_{20}(z)}\right) \partial_{\psi}+4 \omega^{2}\left\{\left(\alpha_{m}+\frac{1}{2} \alpha_{n}\right)\left(\frac{1}{c_{10}^{2}(z)}+\frac{1}{c_{20}^{2}(z)}\right)\right. \\
\left.-\frac{2}{c_{10}(z) c_{20}(z)}\left(\tilde{\alpha}_{m}+\frac{1}{2} \tilde{\alpha}_{n} \mathbf{1}_{\left\{\tau_{1}=\tau_{2}\right\}}(z) \cos \psi\right)\right\} \partial_{\psi}^{2}
\end{array}
$$

whose coefficients depend on the following media statistics

$$
\begin{aligned}
\alpha_{m} & =\int_{0}^{\infty} \mathbb{E}\left\{m_{1}(s) m_{1}(0)\right\} \mathrm{d} s=\int_{0}^{\infty} \mathbb{E}\left\{m_{2}(s) m_{2}(0)\right\} \mathrm{d} s \\
\tilde{\alpha}_{m} & =\int_{0}^{\infty} \mathbb{E}\left\{m_{1}(s) m_{2}(0)\right\} \mathrm{d} s=\int_{0}^{\infty} \mathbb{E}\left\{m_{2}(s) m_{1}(0)\right\} \mathrm{d} s \\
\alpha_{n} & =\int_{0}^{\infty} \mathbb{E}\left\{n_{1}(s) n_{1}(0)\right\} \mathrm{d} s=\int_{0}^{\infty} \mathbb{E}\left\{n_{2}(s) n_{2}(0)\right\} \mathrm{d} s \\
\tilde{\alpha}_{n} & =\int_{0}^{\infty} \mathbb{E}\left\{n_{1}(s) n_{2}(0)\right\} \mathrm{d} s=\int_{0}^{\infty} \mathbb{E}\left\{n_{2}(s) n_{1}(0)\right\} \mathrm{d} s
\end{aligned}
$$

where

$$
m_{j}(s)=\frac{\mu_{j}(s)+\eta_{j}(s)}{2}, \quad n_{j}(s)=\frac{\mu_{j}(s)-\eta_{j}(s)}{2}, \quad j=1,2 .
$$


Furthermore, $\mathbf{1}_{\left\{\tau_{1}=\tau_{2}\right\}}(\cdot)$ denotes the indicator function of the set $\left\{z \leq 0: \tau_{1}(z)=\right.$ $\left.\tau_{2}(z)\right\}$ where

$$
\tau_{j}(z)=\int_{z}^{0} \frac{\mathrm{d} s}{c_{j 0}(s)}, \quad j=1,2,
$$

represent travel time from location $z$ to the interface in the corresponding background medium.

Let $W_{z}$, be a standard one-dimensional backward Brownian motion with $z \in$ $(-\infty, 0]$ defined on a complete probability space (i.e. $W_{-z}$ is a standard onedimensional Brownian motion) [15]. Consider the second order backward Itô stochastic partial differential equation

$$
\mathrm{d} w+\left(\mathcal{L}_{z} w\right) \mathrm{d} z+2 \omega \sqrt{\gamma_{m}(z)} \partial_{\psi} w \overleftarrow{\mathrm{d}} W_{z}=0, \quad \text { for } z<0
$$

with final condition

$$
\left.w\right|_{z=0}=\mathrm{e}^{\mathrm{i} \psi},
$$

where $\overleftarrow{\mathrm{d}} W_{z}$ represents the backward Itô's differential of $W_{z}[\mathbf{1 5}, \mathbf{1 6}]$ and

$$
\gamma_{m}(z)=2\left\{\alpha_{m}\left(\frac{1}{c_{10}^{2}(z)}+\frac{1}{c_{20}^{2}(z)}\right)-2 \frac{\tilde{\alpha}_{m}}{c_{10}(z) c_{20}(z)}\right\}
$$

From $[\mathbf{1 5}, \mathbf{1 6}]$, we know that the stochastic equation (A.5) has a unique solution $w(z, \psi ; \omega, h)$, which is a backward semimartingale. Let us define

$$
\tilde{w}_{12}(\omega, h)=\lim _{z \rightarrow-\infty} w(z, \psi ; \omega, h)
$$

and set

$$
\begin{array}{r}
\left.\widehat{\Lambda_{12}(\omega,} \cdot\right)(h)=\tilde{w}_{12}(\omega, h), \\
\widehat{K_{12, t_{0}}^{\mathrm{TR}}}(\omega)=\left(\Lambda_{12}(\omega, \cdot) \star G_{t_{0}}(-\cdot)\right)(0) .
\end{array}
$$

Notice that in general these are random functions. In the important case where $G_{t_{0}}(\cdot)=\mathbf{1}_{\left[0, t_{0}\right]}(\cdot)$ (the indicator function of the interval $\left[0, t_{0}\right]$ ) (A.7) simplifies to

$$
\widehat{K_{12, t_{0}}^{\mathrm{TR}}}(\omega)=\int_{0}^{t_{0}} \Lambda_{12}(\omega, s) \mathrm{d} s .
$$

In deriving this result, we first establish the tightness of this family of timereversed signals to ensure that the limit exist. Then, using a diffusion-approximation theorem, we are able to characterize the limit of the corresponding finite-dimensional distributions by determining all their associated statistical moments. Finally, using Itô formula one arrives at the representation above. We notice that if a multiscale model for the random media fluctuations is used, then the statistics (A.4) will smoothly depend on the depth $z$.

We next make some remarks about the stochastic equation (A.5). Note that it is not stochastic when $\gamma_{m}=0$, a condition which is fulfilled if and only if $c_{10}(\cdot)=c_{20}(\cdot)$ and $\rho_{m} \equiv \tilde{\alpha}_{m} / \alpha_{m}=1$.

It is worth noticing that when the time reversal is performed in an unchanged medium, the conditions above are satisfied, and we recover the following well-known results [7]: In the unchanged case, the time-reversed signal is deterministic (thus 
statistically stable) and the corresponding (deterministic) refocusing kernel is described as follows

$$
\begin{aligned}
& \widehat{K_{12, t_{0}}^{\mathrm{TR}}}(\omega)=\widehat{K_{t_{0}}^{\mathrm{TR}}}(\omega)=\int \Lambda(\omega, s) G_{t_{0}}(s) \mathrm{d} s, \\
& \widehat{\Lambda(\omega, \cdot)}(h)=\tilde{w}(\omega, h)=\lim _{z \rightarrow-\infty} w(z, \psi ; \omega, h) .
\end{aligned}
$$

The function $w(z, \psi ; \omega, h)$ satisfies the backward Kolmogorov partial differential equation

$$
\frac{\partial w}{\partial t}+\mathcal{L}_{z} w=0, \quad \text { for } z<0
$$

with final condition

$$
\left.w\right|_{z=0}=\mathrm{e}^{\mathrm{i} \psi},
$$

and the partial differential operator $\mathcal{L}_{z}$ is given by

$$
\mathcal{L}_{z}=\frac{2 h}{c_{0}(z)} \partial_{\psi}+\frac{4 \omega^{2} \alpha_{n}}{c_{0}^{2}(z)}(1-\cos \psi) \partial_{\psi}^{2}
$$

where $c_{0}(\cdot)=c_{10}(\cdot)=c_{20}(\cdot)$.

Furthermore, for low frequencies $\omega$, in the case where $G_{t_{0}}(\cdot)=\mathbf{1}_{\left[0, t_{0}\right]}(\cdot)$, the refocusing kernel has the following asymptotic behavior (see for instance [12])

$$
\widehat{K_{t_{0}}^{\mathrm{TR}}}(\omega) \approx \Gamma_{n} \omega^{2}
$$

where

$$
\Gamma_{n}=2 \alpha_{n} \int_{0}^{t_{0} / 2} \frac{\mathrm{d} \tau}{\tilde{c}_{0}(\tau)}
$$

and $\tilde{c}_{0}(\cdot)$ represents the sound speed $c_{0}(\cdot)$ considered as a function of travel time.

Next, we shall focus on the case of two media. Let

$$
Z_{0}= \begin{cases}\sup \left\{z \leq 0: c_{10}(z) \neq c_{20}(z)\right\}, & \text { if } \rho_{m}=1 \\ 0, & \text { if } \rho_{m} \neq 1\end{cases}
$$

with the understanding that if the set over which we take the supremum happens to be empty we put $Z_{0}=-\infty$. In this particular case (i.e. when $Z_{0}=-\infty$ ) we have that the refocused pulse is statistically stable, this is related to the fact that the propagation velocity remains unperturbed as was remarked in [14]. We refer to the interval $\left[Z_{0}, 0\right]$ as the unperturbed propagation velocity region (or slab). As a remark, we notice that for a multi-scale model of the random media fluctuations the coefficient $\rho_{m}$ smoothly depends on the depth $z$, thus the definition of $Z_{0}$ shall be changed to $\sup \left\{z \leq 0: c_{10}(z) \neq c_{20}(z)\right.$ or $\left.\rho_{m}(z) \neq 1\right\}$.

Observe that the factor $\mathbf{1}_{\left\{\tau_{1}=\tau_{2}\right\}}(z)$ in (A.3) switches on and off the dependence of $\mathcal{L}_{z}$ on $\psi$, in particular if

$$
Z_{1}=\inf \left\{z \leq 0: \tau_{1}(z)=\tau_{2}(z)\right\}>-\infty
$$

one can explicitly find $w(z, \psi ; \omega, h)$ for $z<Z_{1}$ as a function of $w\left(Z_{1}^{+}, \psi ; \omega, h\right)$. Furthermore, we obtain that

$$
\tilde{w}_{12}(\omega, h)=\frac{1}{2 \pi} \int_{0}^{2 \pi} w\left(Z_{1}^{+}, \psi ; \omega, h\right) \mathrm{d} \psi
$$

In particular, if $Z_{1}=0$, we have that $\tilde{w}(\omega, h)=0$ so the refocused pulse is the null signal. This is an extreme situation in which the travel time difference in the 
forward and backward propagation generates fast phases that ultimately annihilates the time-reversed reflected pulse. We called the interval $\left(-\infty, Z_{1}\right]$ the asynchronous travel time region (or slab).

A.1.1. Statistically stable refocusing. It should be noted that statistical stability means that the limiting time reversed reflected signal (A.2) is deterministic and therefore the convergence occurs in probability. We now discuss an interesting situation in which we have a statistically stable refocusing.

Note that $-\infty \leq Z_{1} \leq Z_{0} \leq 0$. Suppose that $Z_{0}=Z_{1}$, i.e. the unperturbed velocity and asynchronous travel time regions complement each other, then from the observations above we have that under this condition the refocusing is statistically stable. Indeed, from the definition of $Z_{0}$ we get that $w\left(Z_{0}, \psi\right)=w\left(Z_{1}^{+}, \psi\right)$ is a deterministic function, thus from (A.14) and the representation given by (A.7) the result follows.

This is a very interesting situation in which the statistical stability comes from the fact that the propagation velocity remains unperturbed down to some depth below which the fast phase associated with the travel time difference kills out the effect of velocity perturbations.

This occurs for instance if $\rho_{m}=1$ and $\delta c=c_{20}-c_{10} \geq 0$ (or $\left.\leq 0\right), \operatorname{supp} \delta c=$ $\left[Z_{1}^{\prime}, Z_{0}^{\prime}\right]$ (or $\operatorname{supp} \delta c=\left(-\infty, Z_{0}^{\prime}\right]$ ). In this case we say that the medium is changed by increasing (decreasing) the propagation velocity. We are specially interested in the case where $\delta c$ is compactly supported as a model for the analysis of inclusion effects.

We remark that in the statistically stable case, for instance under the conditions stated before, we have convergence in probability whereas in the general situation the convergence occurs in distribution. This means that in the former case the refocused signal (for a small $\varepsilon$ ) remains close to the limiting deterministic signal (described by equations (A.5)-(A.7) and (A.2)) with high probability.

Next, we continue to study the solution of (A.5) and its relationship with the refocusing kernel (A.7).

A.1.2. Stochastic transport equations and the (random) refocusing kernel. We proceed by solving equation (A.5) using a Fourier series in $\psi$

$$
w(z, \psi ; \omega, h)=\sum_{N=-\infty}^{\infty} V^{N} \mathrm{e}^{\mathrm{i} N \psi}
$$

We obtain a system of backward stochastic differential equations for the coefficients $V^{N}$ for $N \geq 0$

$$
\begin{aligned}
\mathrm{d} V^{N}+\left\{\frac{2 \mathrm{i} h N}{\bar{c}_{0}(z)} V^{N}+2 \omega^{2} \beta_{n}(z)\left[(N+1)^{2} V^{N+1}+(N-1)^{2} V^{N-1}\right]\right. \\
\left.-2 \omega^{2} \beta_{m n}(z) N^{2} V^{N}\right\} \mathrm{d} z+2 \mathrm{i} \omega \sqrt{\gamma_{m}(z)} N V^{N \overleftarrow{\mathrm{d}} W_{z}=0},
\end{aligned}
$$

for $z<0$ with the final conditions

$$
\left.V^{N}\right|_{z=0}=\delta_{N, 1}
$$


where $\delta_{M, S}$ represents the Kronecker delta and

$$
\begin{aligned}
\frac{1}{\bar{c}_{0}(z)} & =\frac{1}{2}\left(\frac{1}{c_{10}(z)}+\frac{1}{c_{20}(z)}\right), \\
\beta_{n}(z) & =\frac{\tilde{\alpha}_{n}}{c_{10}(z) c_{20}(z)} \mathbf{1}_{\left\{\tau_{1}=\tau_{2}\right\}}(z), \\
\beta_{m n}(z) & =\gamma_{m}(z)+\alpha_{n}\left(\frac{1}{c_{10}^{2}(z)}+\frac{1}{c_{20}^{2}(z)}\right) .
\end{aligned}
$$

Furthermore, for $N<0$ one gets that $V^{N}=0$, and we finally have that

$$
\tilde{w}_{12}(\omega, h)=\lim _{z \rightarrow-\infty} V^{0}(z ; \omega, h) .
$$

Introducing the inverse Fourier transform

$$
U^{N}(z, t, \omega)=\frac{1}{2 \pi} \int \mathrm{e}^{\mathrm{i} h t} V^{N}(z ; \omega, h) \mathrm{d} h, \quad \text { for } N \geq 0,
$$

and the averaged travel time $\tau=\left(\tau_{1}(z)+\tau_{2}(z)\right) / 2$ as a new coordinate, we obtain the stochastic transport equations

$$
\begin{aligned}
\mathrm{d} U^{N}+2 N \frac{\partial U^{N}}{\partial t} \mathrm{~d} \tau=2 \omega^{2} & \left\{\vartheta_{n}(\tau)\left[(N+1)^{2} U^{N+1}+(N-1)^{2} U^{N-1}\right]\right. \\
& \left.-\vartheta_{m n}(\tau) N^{2} U^{N}\right\} \mathrm{d} \tau+2 \mathrm{i} \omega \sqrt{\bar{\gamma}_{m}(\tau)} N U^{N} \mathrm{~d} M_{\tau}
\end{aligned}
$$

for $\tau>0, N \geq 0$, with $U^{-1}=0$ and the initial conditions

$$
\left.U^{N}\right|_{\tau=0}=\delta_{N, 1} \delta(t) .
$$

The coefficients are given by

$$
\begin{aligned}
\bar{\gamma}_{m}(\tau) & =\gamma_{m}(\xi(\tau)) \\
\vartheta_{n}(\tau) & =\bar{c}_{0}(\xi(\tau)) \beta_{n}(\xi(\tau)) \\
\vartheta_{m n}(\tau) & =\bar{c}_{0}(\xi(\tau)) \beta_{m n}(\xi(\tau))
\end{aligned}
$$

where $\xi(\tau)$ represents the inverse function of the averaged travel time, $\mathrm{d} M_{\tau}$ the Itô differential of the (forward) martingale $M_{\tau}=W_{-\xi(\tau)}$ and $\delta(t)$ the Dirac $\delta$-function.

This is a system of stochastic hyperbolic equations, reflecting the fact that the pulse propagates with a finite speed. As a consequence, we have that

$$
\Lambda_{12}(t, \omega)=U^{0}\left(\tau^{\prime}, t, \omega\right)
$$

for any $\tau^{\prime} \geq \frac{t}{2}$. On the other hand, from (A.14) and (A.15) we have that $\tilde{w}(\omega, h)=$ $V^{0}\left(Z_{1}^{+} ; \omega, h\right)$, and consequently

$$
\Lambda_{12}(t, \omega)=U^{0}\left(T_{1}, t, \omega\right)
$$

where $T_{1}=\tau_{1}\left(Z_{1}\right)=\tau_{2}\left(Z_{1}\right)$ is the time required to reach depth $Z_{1}$ and also the time required to get from there back to the interface. Therefore, if the cutoff function $G_{t_{0}}(\cdot)=\mathbf{1}_{\left[0, t_{0}\right]}(\cdot)$ then the refocusing kernel can be written as

$$
\widehat{K_{12, t_{0}}^{\mathrm{TR}}}(\omega)=\int_{0}^{t_{0}} U^{0}\left(T_{1} \wedge \frac{t_{0}}{2}, s, \omega\right) \mathrm{d} s
$$

This means that the refocused pulse does not depend on the media properties below depth $Z_{1}$, regardless of how large the recording time $t_{0}$ is. In particular, when the unperturbed velocity and asynchronous travel time regions complement each other 
(i.e. $Z_{0}=Z_{1}$ ) the refocused pulse does not carry information about the inclusion characteristics.

\section{Appendix B. Asymptotic analysis for time reversal detection}

B.1. Asymptotics of the measured time-reversed difference reflection. We model the error introduced during a direct measurement as an additive 'noise' varying on the scale $\varepsilon^{a}$ with $a>0$, that is the measured signal $g_{\text {meas }}^{\varepsilon}(t)$ associated with the actual signal $g^{\varepsilon}(t)$ is given as

$$
g_{\text {meas }}^{\varepsilon}(t)=g^{\varepsilon}(t)+\nu\left(\frac{t}{\varepsilon^{a}}\right)
$$

where $\nu(\cdot)$ is a mean zero, stationary Gaussian random process with an integrable autocorrrelation function, defined on a certain probability space.

During the time reversal procedure we introduce direct measurement errors three times, during acquisition of the two primary reflected and the refocused signals. We assume that these direct noise sources are statistically independent. Consequently, the measurement error in the whole time reversal procedure is given by the random vector-process $\boldsymbol{\nu}(\cdot)=\left(\nu_{1}(\cdot), \nu_{2}(\cdot), \nu_{3}(\cdot)\right)^{t}$ defined on the corresponding probability space.

Consequently, we have the representations

$$
\nu_{i}(s)=\int \mathrm{e}^{\mathrm{i} \omega s} \Phi_{i}(\mathrm{~d} \omega), \quad i=1,2,3,
$$

where the random spectral measures $\Phi_{i}(\cdot)$ satisfy the relations

$$
E\left\{\Phi_{i}\left(\mathrm{~d} \omega^{\prime}\right) \overline{\Phi_{j}\left(\mathrm{~d} \omega^{\prime \prime}\right)}\right\}=\delta_{i j} \delta\left(\omega^{\prime}-\omega^{\prime \prime}\right) F_{\nu}\left(\omega^{\prime}\right) \mathrm{d} \omega^{\prime}, \quad i, j=1,2,3 .
$$

After some straightforward calculations we get that

$$
\begin{gathered}
B_{t_{0}, \text { meas }}^{\varepsilon, \mathrm{TRD}}(s)=B_{t_{0}}^{\varepsilon, \mathrm{TRD}}(s)+B_{t_{0}, \nu}^{\varepsilon}(s) \\
B_{t_{0}, \nu}^{\varepsilon}(s)=B_{t_{0}, \delta \nu}^{\varepsilon}(s)+\nu_{3}\left(\frac{s}{\varepsilon^{a-1}}\right) .
\end{gathered}
$$

This term is associated with measurements errors and arises from the propagation of the difference of the direct measurements noise related to the primary reflections $\delta \nu(\cdot)=\nu_{2}(\cdot)-\nu_{1}(\cdot)$ and the error in the direct measurement of the time-reversed difference reflection (cf. (B.2)). Furthermore, the primary reflections propagated noise can be written as

$$
B_{t_{0}, \delta \nu}^{\varepsilon}(s)=\frac{1}{2 \pi} \iint \mathrm{e}^{\mathrm{i}\left(\varepsilon^{1-a} \omega-\varepsilon h\right) s} R_{2}^{\varepsilon}\left(\frac{\omega}{\varepsilon^{a-1}}-\varepsilon h\right) \hat{G}_{t_{0}}(h) \Phi_{\delta \nu}(\mathrm{d} \omega) \mathrm{d} h
$$

where $\Phi_{\delta \nu}(\cdot)$ is the random spectral measure given by

$$
\Phi_{\delta \nu}(\cdot)=\Phi_{2}(\cdot)-\Phi_{1}(\cdot) .
$$

We shall determine the limit in distribution of the measured time-reversed difference reflection $B_{t_{0}, \text { meas }}^{\varepsilon, \text { TRD }}(\cdot)$ given by (B.1). We focus on the case where the velocity changes in an increasing/decreasing fashion. Note that in this case $B_{t_{0}}^{\varepsilon, \text { TRD }}(s)$ converges in probability to the deterministic signal $B_{t_{0}}^{\mathrm{TRD}}(\cdot)$ given by $(2.6)$. Thus, taking into account Slutsky's theorem [20], to characterize the limiting measured time-reversed signal it is enough to analyse the asymptotic behaviour of the contribution associated with the measurement noise $B_{t_{0}, \nu}^{\varepsilon}(s)$ given by (B.2). 
We note that for $a \neq 1$, this contribution can be filtered out in the asymptotic limit $\varepsilon \rightarrow 0$. This means, roughly speaking, that the signal $B_{t_{0} \text {, meas }}^{\varepsilon, \text { TRD }}(\cdot)$ is statistically stable, and converges to the deterministic signal $B_{t_{0}}^{\mathrm{TRD}}(\cdot)$ given by $(2.6)$ in this asymptotic regime.

More exactly, we have that the random variable

$$
\left\langle B_{t_{0}, \boldsymbol{\nu}}^{\varepsilon}, \phi\right\rangle=\int B_{t_{0}, \boldsymbol{\nu}}^{\varepsilon}(s) \phi(s) \mathrm{d} s
$$

converges in probability to zero as $\varepsilon \rightarrow 0$, for any function $\phi \in L^{1}(\mathbb{R})$ such that $\hat{\phi}(0)=0$. Since $E\left\{\left\langle B_{t_{0}, \boldsymbol{\nu}}^{\varepsilon}, \phi\right\rangle\right\}=0$, from Chebyshev inequality it is enough to prove that $E\left\{\left|\left\langle B_{t_{0}, \nu}^{\varepsilon}, \phi\right\rangle\right|^{2}\right\} \rightarrow 0$ as $\varepsilon \rightarrow 0$. Taking into account decomposition (B.2) this will follow by establishing that

$$
\lim _{\varepsilon \rightarrow 0} E\left\{\left|\left\langle B_{t_{0}, \delta \nu}^{\varepsilon}, \phi\right\rangle\right|^{2}\right\}=\lim _{\varepsilon \rightarrow 0} E\left\{\left|\left\langle\nu_{3}\left(\cdot / \varepsilon^{a-1}\right), \phi(\cdot)\right\rangle\right|^{2}\right\}=0 .
$$

To prove that $E\left\{\left|\left\langle B_{t_{0}, \delta \nu}^{\varepsilon}, \phi\right\rangle\right|^{2}\right\} \rightarrow 0$ as $\varepsilon \rightarrow 0$, we use the representation

$$
\left\langle B_{t_{0}, \delta \nu}^{\varepsilon}, \phi\right\rangle=\frac{1}{2 \pi} \iint \hat{\phi}\left(\frac{\omega}{\varepsilon^{a-1}}-\varepsilon h\right) R_{2}^{\varepsilon}\left(\frac{\omega}{\varepsilon^{a-1}}-\varepsilon h\right) \hat{G}_{t_{0}}(h) \Phi_{\delta \nu}(\mathrm{d} \omega) \mathrm{d} h,
$$

to get that

$$
\begin{aligned}
E\left\{\left|\left\langle B_{t_{0}, \delta \nu}^{\varepsilon}, \phi\right\rangle\right|^{2}\right\}= & \frac{1}{2 \pi^{2}} \iint \hat{\phi}\left(\frac{\omega}{\varepsilon^{a-1}}-\varepsilon h_{1}\right) \overline{\hat{\phi}}\left(\frac{\omega}{\varepsilon^{a-1}}-\varepsilon h_{2}\right) \hat{G}_{t_{0}}\left(h_{1}\right) \overline{\hat{G}_{t_{0}}}\left(h_{2}\right) \times \\
& \mathbb{E}\left\{R_{2}^{\varepsilon}\left(\frac{\omega}{\varepsilon^{a-1}}-\varepsilon h_{1}\right) \overline{R_{2}^{\varepsilon}}\left(\frac{\omega}{\varepsilon^{a-1}}-\varepsilon h_{2}\right)\right\} F_{\nu}(\omega) \mathrm{d} \omega \mathrm{d} h_{1} \mathrm{~d} h_{2} .
\end{aligned}
$$

Next, assuming that $\hat{G}_{t_{0}} \in L^{1}(\mathbb{R})$, taking into account the boundedness of the reflection coefficient $R_{2}^{\varepsilon}$ and the properties of the function $\hat{\phi}$, the result easily follows from Lebesgue's dominated convergence theorem.

Furthermore, it can be established in a similar way that

$$
E\left\{\left|\left\langle\nu_{3}\left(\cdot / \varepsilon^{a-1}\right), \phi(\cdot)\right\rangle\right|^{2}\right\} \rightarrow 0
$$

as $\varepsilon \rightarrow 0$, to get the convergence of $\left\langle B_{t_{0}, \nu}^{\varepsilon}, \phi\right\rangle$ to zero.

From now on, we focus on the case $a=1$, in which the randomness plays an important role in the asymptotic behaviour of the measured time-reversed difference signal $B_{t_{0}, \text { meas }}^{\varepsilon, \text { TRD }}(\cdot)$.

By using that the random process $\delta \nu(\cdot)$ is stationary, Gaussian and centered and also considering the asymptotics for the moments of the reflection coefficient $R_{2}^{\varepsilon}$ one can establish the convergence in distribution as $\varepsilon \downarrow 0$ of $B_{t_{0}, \delta \nu}^{\varepsilon}(s)$ to a stationary, centred Gaussian process $B_{t_{0}, \delta \nu}(s)$ with covariance function given by

$$
C_{t_{0}, \delta \nu}(s)=2 \int \mathrm{e}^{\mathrm{i} \omega s} \widehat{K_{2, t_{0}}^{\mathrm{R}}}(\omega) F_{\nu}(\omega) \mathrm{d} \omega
$$

where

$$
\begin{array}{r}
\widehat{K_{2, t_{0}}^{\mathrm{R}}}(\omega)=\left(\Lambda_{22}(\omega, \cdot) \star G_{t_{0}}^{2}(-\cdot)\right)(0)=\int \Lambda_{22}(\omega, s) G_{t_{0}}^{2}(s) \mathrm{d} s, \\
\widehat{\Lambda_{22}(\omega, \cdot)}(h)=\tilde{w}_{22}(\omega, h)=\lim _{z \rightarrow-\infty} w(z, \psi ; \omega, h),
\end{array}
$$

and $w(z, \psi)$ satisfies the following backward Kolmogorov partial differential equation

$$
\frac{\partial w}{\partial t}+\mathcal{L}_{z} w=0, \quad \text { for } z<0
$$


with final condition

$$
\left.w\right|_{z=0}=\mathrm{e}^{\mathrm{i} \psi},
$$

and the partial differential operator $\mathcal{L}_{z}$ given by

$$
\mathcal{L}_{z}=\frac{2 h}{c_{20}(z)} \partial_{\psi}+\frac{4 \omega^{2} \alpha_{n}}{c_{20}^{2}(z)}(1-\cos \psi) \partial_{\psi}^{2} .
$$

Furthermore, since $\delta \nu(\cdot)$ and $\nu_{3}(\cdot)$ are statistically independent we finally get that $B_{t_{0}, \nu}^{\varepsilon}(s)$ converges in distribution as $\varepsilon \downarrow 0$ to a mean zero, stationary, Gaussian random process with a covariance function given by

$$
C_{t_{0}, \boldsymbol{\nu}}(s)=\int \mathrm{e}^{\mathrm{i} \omega s}\left(1+2 \widehat{K_{2, t_{0}}^{\mathrm{R}}}(\omega)\right) F_{\nu}(\omega) \mathrm{d} \omega .
$$

Finally, from the Slutsky's theorem $[\mathbf{2 0}]$, it follows that $B_{t_{0}, \text { meas }}^{\varepsilon, \text { TRD }}(s)$ converges in distribution as $\varepsilon \downarrow 0$ to a Gaussian random process with mean $B_{t_{0}}^{\mathrm{TRD}}(s)$ given by (2.6) and covariance function given by (B.7).

We remark that for time reversal in a random medium which remains fixed, a similar analysis of the measured refocused pulse yields the convergence in distribution to a Gaussian random process whose mean is the limiting deterministic refocused signal and the covariance function is similar to (B.7) except for the prefactor 2. Moreover, for time reversal in a generally changing medium a similar result holds as long as the limiting refocused signal (when no error measurements are present) is deterministic.

B.2. Analysis of the statistical test. Recall that the detection problem can be stated as a hypothesis testing problem corresponding to

$\mathrm{H}_{0}: \mathbf{x}$ is a sample of the random variable $\mathbf{X}_{0} \sim \mathcal{N}\left(\mathbf{0}, \mathbf{C}_{0}\right)$

$\mathrm{H}_{\mathrm{a}}: \mathbf{x}$ is a sample of a random variable $\mathbf{X}_{a} \sim \mathcal{N}\left(\boldsymbol{\mu}_{\mathrm{a}}, \mathbf{C}_{\mathrm{a}}\right)$.

The mean vector $\boldsymbol{\mu}_{\mathrm{a}}=\left(B_{t_{0}}^{\mathrm{TRD}}\left(s_{1}\right), \cdots, B_{t_{0}}^{\mathrm{TRD}}\left(s_{M}\right)\right)^{t}$ with the $B_{t_{0}}^{\mathrm{TRD}}(\cdot)$ given by (2.6). Furthermore, the covariance matrices $\mathbf{C}_{0}, \mathbf{C}_{a}$ are symmetric Toeplitz matrices with entries

$$
\begin{aligned}
& \mathbf{C}_{0, i j}=\frac{1}{2 \pi} \int_{-\pi}^{\pi} \mathrm{e}^{\mathrm{i}(j-i) \lambda} F_{0}(\lambda) \mathrm{d} \lambda \\
& \mathbf{C}_{a, i j}=\frac{1}{2 \pi} \int_{-\pi}^{\pi} \mathrm{e}^{\mathrm{i}(j-i) \lambda} F_{\mathrm{a}}(\lambda) \mathrm{d} \lambda
\end{aligned}
$$

where

$$
\begin{aligned}
& F_{\mathrm{a}}(\lambda)=\frac{2 \pi}{h} \sum_{k=-\infty}^{\infty} F_{\nu}\left(\frac{\lambda+2 k \pi}{h}\right)\left(1+2 \widehat{K_{2, t_{0}}^{\mathrm{R}}}\left(\frac{\lambda+2 k \pi}{h}\right)\right) \\
& F_{0}(\lambda)=\frac{2 \pi}{h} \sum_{k=-\infty}^{\infty} F_{\nu}\left(\frac{\lambda+2 k \pi}{h}\right)\left(1+2 \widehat{K_{1, t_{0}}^{\mathrm{R}}}\left(\frac{\lambda+2 k \pi}{h}\right)\right)
\end{aligned}
$$

with $K_{2, t_{0}}^{\mathrm{R}}(\cdot)$ given by equations (B.4)-(B.6) and $K_{1, t_{0}}^{\mathrm{R}}(\cdot)$ corresponding to analogous equations but with the differential operator $\mathcal{L}_{z}$ in (B.6) depending on the sound speed $c_{10}(\cdot)$.

In order to explicitly write the dependence of the covariance matrices on the functions $F_{0}(\cdot)$ and $F_{\mathrm{a}}(\cdot)$, we set $\mathbf{C}_{0}=T_{M}\left(F_{0}\right)$ and $\mathbf{C}_{\mathrm{a}}=T_{M}\left(F_{\mathrm{a}}\right)$. 
Let us introduce the function

$$
F(\lambda)=\frac{2 \pi}{h} \sum_{k=-\infty}^{\infty} F_{\nu}\left(\frac{\lambda+2 k \pi}{h}\right)
$$

and its extreme values $m_{F}=\operatorname{essinf} F, M_{F}=\operatorname{ess} \sup F$. Since $F_{\nu}(\lambda) \geq 0$ and $0 \leq \widehat{K_{t_{0}, 2}^{\mathrm{R}}}(\lambda) \leq 1$, we get that

$$
\operatorname{essinf} F_{\mathrm{a}}=m_{F_{\mathrm{a}}} \geq m_{F}, \quad \operatorname{ess} \sup F_{\mathrm{a}}=M_{F_{\mathrm{a}}} \leq 3 M_{F} .
$$

Consequently, the corresponding Toeplitz determinant $\left|T_{M}\left(F_{\mathrm{a}}\right)\right|$ satisfies the estimates

$$
\left(m_{F}\right)^{M} \leq\left|T_{M}\left(F_{\mathrm{a}}\right)\right| \leq\left(3 M_{F}\right)^{M} .
$$

In what follows, we assume that $m_{F}>0$.

In general, the covariance matrix $\mathbf{C}_{0}$ is unknown, however it can be estimated by performing a time reversal experiment in the unchanged medium. (In the case of a homogeneous medium it can be explicitly computed from equation (B.3).) Thus, we now assume that $\mathbf{C}_{0}$ is given, or equivalently that we know $F_{0}$. Concerning the covariance matrix $\mathbf{C}_{\mathrm{a}}$, since it is completely characterized by $F_{\mathrm{a}}$, we assume that a set of admissible functions $\mathcal{F}$ is given.

We can reformulate the problem as follows: given a sample $\mathbf{x}$ of a random variable distributed as $\mathcal{N}(\boldsymbol{\mu}, \mathbf{C})$ test the hypotheses $\mathrm{H}_{0}$ vs. $\mathrm{H}_{\mathrm{a}}$, where

- $\mathrm{H}_{0}: \boldsymbol{\mu}=\mathbf{0}$ and $\mathbf{C}=\mathbf{C}_{0}$

- $\mathrm{H}_{\mathrm{a}}: \boldsymbol{\mu} \neq \mathbf{0}$ and $\mathbf{C}=T_{M}(\tilde{F})$ with $\tilde{F} \in \mathcal{F}$.

The set $\mathcal{F}$ consists of the functions that can be represented as in (B.8), where the kernel $K_{2, t_{0}}^{\mathrm{R}}$ corresponds to an admissible random medium through the relations (B.4)-(B.6). Here we consider the set of functions $\tilde{F}$ satisfying bounds similar to $F_{\mathrm{a}}$ in $(\mathrm{B} .9)$.

B.2.1. The Likelihood Ratio Test. When testing composite hypotheses, as in the situation at hand, a useful way for selecting the rejection region is to use the Likelihood Ratio (LR) Test [21]. We consider it as a starting point but later on, after some asymptotic analysis we shall slightly modify this test in order to achieve a better performance.

The let the rejection region for this test at significance level $\alpha$ is given by $R_{\alpha}=\left\{\mathbf{x}: \Gamma(\mathbf{x}) \geq c_{\alpha}\right\}$ where

$$
\Gamma(\mathbf{x})=\frac{\sup _{\boldsymbol{\mu} \in \mathbb{R}^{M}, \tilde{F} \in \mathcal{F}}\left|T_{M}(\tilde{F})\right|^{-1 / 2} \exp \left\{-\frac{1}{2}(\mathbf{x}-\boldsymbol{\mu})^{t} T_{M}^{-1}(\tilde{F})(\mathbf{x}-\boldsymbol{\mu})\right\}}{\left|\mathbf{C}_{0}\right|^{-1 / 2} \exp \left\{-\frac{1}{2} \mathbf{x}^{t} \mathbf{C}_{0}^{-1} \mathbf{x}\right\}},
$$

and $c_{\alpha}$ is determined from the equation $\operatorname{Pr}\left\{\mathbf{x} \in R_{\alpha} \mid H_{0}\right\}=\alpha$. After some simple algebra, we arrive at the test statistic $Q_{M}(\mathbf{x})=\mathbf{x}^{t} \mathbf{C}_{0}^{-1} \mathbf{x}$ and get that $R_{\alpha}=\{\mathbf{x}$ : $\left.Q_{M}(\mathbf{x}) \geq \chi_{M}^{2}(1-\alpha)\right\}$, where $\chi_{M}^{2}(\cdot)$ represents the inverse of the cumulative $\chi^{2}$ distribution function with $M$ degrees of freedom. This is a consequence of the boundedness of $\left|T_{M}(\tilde{F})\right|$ and the fact that under $H_{0}, Q_{M}(\mathbf{x})$ has a $\chi^{2}$-distribution with $M$ degrees of freedom.

In order to measure the performance of the test, we now have to determine its power as a function of the significance level, i.e. the probability of rejection under the alternate hypothesis for each value of $\alpha$. Since the alternate hypothesis is composite the power of the test is parameterized by the mean vector $\boldsymbol{\mu}$ and the covariance matrix $\mathbf{C}=T_{M}(\tilde{F})$ for some $\tilde{F} \in \mathcal{F}$. We find $P(\alpha ; \boldsymbol{\mu}, \mathbf{C})=\operatorname{Pr}\left\{\mathbf{x}^{t} \mathbf{C}_{0}^{-1} \mathbf{x} \geq\right.$ 
$\left.\chi_{M}^{2}(1-\alpha) \mid \mathbf{x} \sim \mathcal{N}(\boldsymbol{\mu}, \mathbf{C})\right\}$. This is the complement of the cumulative distribution function for a quadratic form of a normally distributed random variable, and we have $[\mathbf{2 2}]$ that $P(\alpha ; \boldsymbol{\mu}, \mathbf{C})=G\left(\chi_{M}^{2}(1-\alpha) ; \boldsymbol{\lambda}, \boldsymbol{\xi}\right)$. The function $G(\cdot)$ corresponds to the complement of the cumulative distribution function of the random variable $\sum_{j=1}^{M} \lambda_{j}\left(W_{j}-\xi_{j}\right)^{2}$ where the $W_{j}$ 's are mutually independent $\mathcal{N}(0,1)$ random variables, the $\lambda_{j}$ 's are the eigenvalues of the matrix $\mathbf{C C}_{0}^{-1}$, the vector $\boldsymbol{\xi}=\mathbf{O}^{t} \mathbf{L}^{-1} \boldsymbol{\mu}$, $\mathbf{L}$ is the lower triangular matrix in the Cholesky decomposition of $\mathbf{C}$ and $\mathbf{O}$ is an orthogonal matrix formed by the eigenvectors of $\mathbf{L}^{t} \mathbf{C}_{0}^{-1} \mathbf{L}$. Furthermore, we have the following integral representation

$$
G(q ; \boldsymbol{\lambda}, \boldsymbol{\xi})=\int_{-\mathrm{i} \infty}^{+\mathrm{i} \infty} \frac{\exp (-q u+\phi(u))}{u} \mathrm{~d} u
$$

where the path of integration is indented toward the right at $u=0$, and we have set

$$
\phi(u)=\frac{1}{2} \sum_{j=1}^{M}\left\{\xi_{j}^{2}\left(\frac{1}{1-2 u \lambda_{j}}-1\right)-\log \left(1-2 u \lambda_{j}\right)\right\} .
$$

This integral can be efficiently evaluated with a high accuracy by using a GaussChebyshev quadrature formula $[\mathbf{2 3}]$.

B.2.2. Asymptotics of the LR test statistic. In this section we study the asymptotic behaviour for large $M$ of the scaled test statistic $\tilde{Q}_{M}(\mathbf{x})=Q_{M}(\mathbf{x}) / M$ when $\mathbf{x} \sim \mathcal{N}(\boldsymbol{\mu}, \mathbf{C})$.

The mean and variance of $\tilde{Q}_{M}$ are given by

$$
\begin{aligned}
& \tilde{\mu}_{Q, M}=\frac{1}{M}\left\{\operatorname{Tr}\left(\mathbf{C C}_{0}^{-1}\right)+\boldsymbol{\mu}^{t} \mathbf{C}_{0}^{-1} \boldsymbol{\mu}\right\}=\frac{1}{M} \sum_{j=1}^{M} \lambda_{j}\left(1+\xi_{j}^{2}\right), \\
& \tilde{\sigma}_{Q, M}^{2}=\frac{2}{M^{2}}\left\{\operatorname{Tr}\left(\mathbf{C C}_{0}^{-1}\right)^{2}+2 \boldsymbol{\mu}^{t} \mathbf{C}_{0}^{-1} \mathbf{C C}_{0}^{-1} \boldsymbol{\mu}\right\}=\frac{2}{M^{2}} \sum_{j=1}^{M} \lambda_{j}^{2}\left(1+2 \xi_{j}^{2}\right) .
\end{aligned}
$$

Consider the normalized statistics $z_{M}=\left(\tilde{Q}_{M}-\tilde{\mu}_{Q, M}\right) / \tilde{\sigma}_{Q, M}$. We claim that the random variable $z_{M}$ is asymptotically normally distributed as $\mathcal{N}(0,1)$ for large $M$.

The characteristic function $\psi_{M}(u)=\mathbb{E}\left\{\mathrm{e}^{\mathrm{i} u z_{M}}\right\}$ is given by

$$
\log \psi_{M}(u)=\frac{1}{2} \sum_{j=1}^{M}\left\{\xi_{j}^{2}\left(\frac{1}{1-2 \mathrm{i} \tilde{u} \tilde{\lambda}_{j}}-1\right)-\log \left(1-2 \mathrm{i} \tilde{u} \tilde{\lambda}_{j}\right)\right\}-\mathrm{i} \tilde{u} \tilde{\mu}_{Q, M}
$$

where $\tilde{u}=\frac{u}{\tilde{\sigma}_{Q, M}}$ and $\tilde{\lambda}_{j}=M^{-1} \lambda_{j}$. Furthermore, we have the following estimate

$$
\begin{array}{r}
\log \psi_{M}(u)=\frac{u^{2}}{2}+r_{M}, \\
\left|r_{M}\right| \leq A\left|\frac{u}{\tilde{\sigma}_{Q, M}}\right|^{3} \sum_{j=1}^{M}\left(1+\xi_{j}^{2}\right) \tilde{\lambda}_{j}^{3} .
\end{array}
$$

where the constant $A$ does not depend on $M$. Consequently, it is enough to prove that $r_{M} \rightarrow 0$ as $M \rightarrow+\infty$.

Since, the covariance matrices are Toeplitz matrices, we get the following (uniformly in $M$ ) bounds for the eigenvalues of $\mathbf{C C}_{0}^{-1}[\mathbf{2 4}]$

$$
0<\frac{m_{F}}{3 M_{F}} \leq \operatorname{essinf}\left(\tilde{F} / F_{0}\right) \leq \lambda_{j} \leq \operatorname{ess} \sup \left(\tilde{F} / F_{0}\right) \leq \frac{3 M_{F}}{m_{F}}<+\infty .
$$


As a consequence, one has the estimates

$$
\tilde{\sigma}_{Q, M}^{2} \geq \frac{2}{M^{2}} \sum_{j=1}^{M} \lambda_{j}^{2} \geq \frac{A_{1}}{M} .
$$

Moreover, one can obtain uniform bounds similar to (B.11), for the eigenvalues of the covariance matrices $\mathbf{C}_{0}$ and $\mathbf{C}$. Consequently, we get the estimates

$$
\begin{aligned}
\sum_{j=1}^{M}\left(1+\xi_{j}^{2}\right) \tilde{\lambda}_{j}^{3} & \leq \frac{A_{2}}{M^{2}}+\frac{\left|\boldsymbol{\mu}^{t} \mathbf{C}_{0}^{-1}\left(\mathbf{C C}_{0}^{-1}\right)^{2} \boldsymbol{\mu}\right|}{M^{3}} \leq \frac{A_{2}}{M^{2}}+\frac{\left\|\mathbf{C}_{0}^{-1} \boldsymbol{\mu}\right\|\left\|\left(\mathbf{C C}_{0}^{-1}\right)^{2} \boldsymbol{\mu}\right\|}{M^{3}} \\
& \leq \frac{A_{2}}{M^{2}}+\frac{A_{3}\|\boldsymbol{\mu}\|^{2}}{M^{3}} \leq \frac{A_{2}+A_{3}\|\boldsymbol{\mu}\|_{\infty}^{2}}{M^{2}} \leq \frac{A_{2}^{\prime}}{M^{2}} .
\end{aligned}
$$

Thus, we have that $\left|r_{M}\right| \leq A^{\prime}|u|^{3} M^{-1 / 2}$ and the claim follows.

Next, we focus on the asymptotic behaviour of the power of the test. Let us assume that $\sum_{j=1}^{M} \mu_{j}^{2}<\infty$ uniformly in $M$ and the set of admissible functions $\mathcal{F}$ is contained within the Wiener class (in other words the series $\sum_{j=0}^{\infty} C_{t_{0}, \nu}(j h)$ are absolutely convergent). Applying Szegö's theorem on the distribution of eigenvalues of Toeplitz matrices [24], one gets that

$$
\begin{aligned}
& \tilde{\mu}_{Q, M}=\frac{1}{2 \pi} \int_{-\pi}^{\pi} \frac{\tilde{F}(s)}{F_{0}(s)} \mathrm{d} s+\mathrm{O}\left(M^{-1}\right)=\left\langle\tilde{F} / F_{0}\right\rangle+\mathrm{O}\left(M^{-1}\right), \\
& \tilde{\sigma}_{Q, M}^{2}=\frac{1}{M \pi} \int_{-\pi}^{\pi}\left[\frac{\tilde{F}(s)}{F_{0}(s)}\right]^{2} \mathrm{~d} s+\mathrm{O}\left(M^{-2}\right)=2 M^{-1}\left\langle\left(\tilde{F} / F_{0}\right)^{2}\right\rangle+\mathrm{O}\left(M^{-2}\right) .
\end{aligned}
$$

Recall, that $\chi_{M}^{2}(1-\alpha)=\frac{1}{2}\left(\Phi^{-1}(1-\alpha)+\sqrt{2 M-1}\right)^{2}+\mathrm{o}(1)$ as $M \rightarrow+\infty$, where $\Phi(\cdot)$ represents the cumulative distribution function of a standard normal random variable. Hence, we have that

$$
\begin{aligned}
P(\alpha ; \boldsymbol{\mu}, \mathbf{C}) & =\operatorname{Pr}\left\{z_{M} \geq \frac{M^{-1} \chi_{M}^{2}(1-\alpha)-\tilde{\mu}_{Q, M}}{\tilde{\sigma}_{Q, M}}\right\} \\
& \approx 1-\Phi\left(y_{M}\right),
\end{aligned}
$$

where

$$
y_{M}=\sqrt{\frac{M}{2}}\left(\frac{1-\left\langle\tilde{F} / F_{0}\right\rangle}{\left\langle\left(\tilde{F} / F_{0}\right)^{2}\right\rangle^{\frac{1}{2}}}\right)+\frac{\Phi^{-1}(1-\alpha)}{\left\langle\left(\tilde{F} / F_{0}\right)^{2}\right\rangle^{\frac{1}{2}}}+\mathrm{O}\left(M^{-\frac{1}{2}}\right) .
$$

Therefore, for a fixed significance level $\alpha$, when $\left\langle\tilde{F} / F_{0}\right\rangle>1$ we have that $P(\alpha ; \boldsymbol{\mu}, \mathbf{C}) \rightarrow$ 1 as $M \rightarrow+\infty$. Moreover, asymptotically the rate of convergence does not depend on the measurement noise intensity nor the time-reversed signal energy.

Unfortunately, in the case where $\left\langle\tilde{F} / F_{0}\right\rangle<1$ the test does not behave well, for $M$ large its power approaches zero. In particular, this shows that for a large $M$ the MLR test is biased.

B.2.3. The two-sided LR test. In order to remedy this problem we slightly modify this test by introducing two-tailed rejection regions

$$
\tilde{R}_{\alpha}=\left\{\mathbf{x}: Q_{M}(\mathbf{x}) \leq \chi_{M}^{2}(\alpha / 2) \text { or } Q_{M}(\mathbf{x}) \geq \chi_{M}^{2}(1-\alpha / 2)\right\} .
$$

Now, we have that the power of the modified test

$$
\tilde{P}(\alpha ; \boldsymbol{\mu}, \mathbf{C}) \approx 1+\Phi\left(y_{1, M}\right)-\Phi\left(y_{\mathrm{r}, M}\right),
$$


where

$$
\begin{aligned}
& y_{1, M}=\sqrt{\frac{M}{2}}\left(\frac{1-\left\langle\tilde{F} / F_{0}\right\rangle}{\left\langle\left(\tilde{F} / F_{0}\right)^{2}\right\rangle^{\frac{1}{2}}}\right)+\frac{\Phi^{-1}(\alpha / 2)}{\left\langle\left(\tilde{F} / F_{0}\right)^{2}\right\rangle^{\frac{1}{2}}}+\mathrm{O}\left(M^{-\frac{1}{2}}\right) \\
& y_{\mathrm{r}, M}=\sqrt{\frac{M}{2}}\left(\frac{1-\left\langle\tilde{F} / F_{0}\right\rangle}{\left\langle\left(\tilde{F} / F_{0}\right)^{2}\right\rangle^{\frac{1}{2}}}\right)+\frac{\Phi^{-1}(1-\alpha / 2)}{\left\langle\left(\tilde{F} / F_{0}\right)^{2}\right\rangle^{\frac{1}{2}}}+\mathrm{O}\left(M^{-\frac{1}{2}}\right)
\end{aligned}
$$

as $M \rightarrow+\infty$. Consequently, when $\left\langle\tilde{F} / F_{0}\right\rangle \neq 1$ we get that $\tilde{P}(\alpha ; \boldsymbol{\mu}, \mathbf{C}) \rightarrow 1$. Again, asymptotically the convergence rate does not depend on the noise intensity nor the time-reversed signal energy.

Asymptotically, we are testing whether $\left\langle\tilde{F} / F_{0}\right\rangle$ is equal one or not. This quantity can be interpreted as an average (in frequency space) of the amplification/reduction ratio of the measurement-induced noise in the changed medium to the corresponding noise in the initial medium.

B.2.4. Dependence on the inclusion characteristics. In order to analyze how the characteristics of the inclusion affects the quality of the detection procedure we study their contribution to the leading terms of (B.12).

By assuming that $h$ is sufficiently small, we can disregard the terms with a nonzero $k$ in (B.8) to obtain the approximation

$$
1-\left\langle\tilde{F} / F_{0}\right\rangle \approx \frac{h}{\pi} \int_{-\pi / h}^{\pi / h} \frac{\widehat{K_{1, t_{0}}^{\mathrm{R}}}(\omega)-\widehat{K_{2, t_{0}}^{\mathrm{R}}}(\omega)}{1+2 \widehat{K_{1, t_{0}}^{\mathrm{R}}}(\omega)} \mathrm{d} \omega .
$$

Note that one can get a similar approximation for $\left\langle\left(\tilde{F} / F_{0}\right)^{2}\right\rangle^{1 / 2}$.

Furthermore, assume there is an inclusion that changes the sound speed of the background medium from $c_{1}$ to $c_{2}$, and has size $\Delta z$.

Note that, because of wave localization low frequencies will give a major contribution to the power density of the refocused signal (see for instance [7]). This is also true in the present situation, thus we can use low frequency asymptotic of the refocusing kernel analogous to (A.13) in order to approximate the integral above. We get that

$$
(1-\pi / 4) \Xi \lesssim\left|1-\left\langle\tilde{F} / F_{0}\right\rangle\right| \lesssim \Xi
$$

where $\Xi=\frac{\Delta z}{l} \frac{\left|c_{2}-c_{1}\right|}{c_{2}}$ with $l=c_{1} t_{0} / 2$. Moreover, for $\Xi$ small one also has the approximation $\left\langle\left(\tilde{F} / F_{0}\right)^{2}\right\rangle^{1 / 2} \approx 1$.

We consider the case where $\left\langle\tilde{F} / F_{0}\right\rangle<1$ since the other situation can be treated in the same way. Hence for the power of the test we have that

$$
\begin{aligned}
\tilde{P}(\alpha ; \boldsymbol{\mu}, \mathbf{C}) & \approx 1+\Phi\left(y_{1, M}\right)-\Phi\left(y_{\mathrm{r}, M}\right) \gtrsim \Phi\left(y_{1, M}\right) \\
& \gtrsim \Phi\left(\Xi \sqrt{M / 2}-\Phi^{-1}(1-\alpha / 2)\right) .
\end{aligned}
$$

Finally, it will be better than $1-\tilde{\beta}$ when

$$
M \gtrsim 2\left(\frac{\Phi^{-1}(1-\alpha / 2)+\Phi^{-1}(1-\tilde{\beta})}{\Xi}\right)^{2} .
$$

We remark that this is a rough estimate of the number of sampling points. In the numerical examples presented in section 4, we actually used samples with 
sizes less than half the estimated number of sampling points in order to achieve the desired probability of detection.

\section{References}

1. H. Ammari, J. Garnier and K. Solna, A statistical approach to target detection and localization in the presence of noise, Accepted Waves in Random and Complex Media, 2010.

2. H. Ammari, J. Garnier, H. Kang, W.K. Park and K. Solna, Imaging schemes for perfectly conducting cracks, SIAM Journal on Applied Mathematics, 2010.

3. Borcea, L., Papanicolaou, G. and Tsogka, C., 2005, Interferometric array imaging in clutter. Inverse Problems, 21, 1419-1460.

4. Borcea, L., Papanicolaou, G. and Tsogka, C., 2006, Coherent interferometry in finely layered random mediua. SIAM J. Multiscale Model. Simul., 5, 62-83.

5. Borcea, L., Tsogka, C., Papanicolaou, G. and Berryman, J., 2002, Imaging and time reversal in random media. Inverse Problems, 18, 1247-1279.

6. Bal, G. and Pinaud, O., 2005, Time-reversal-based detection in random media. Inverse Problems, 21(5), 1593-1619.

7. J.P. Fouque, J. Garnier, G. Papanicolaou and K. Sølna, Wave Propagation and Time Reversal in Randomly Layered Media, Springer 2007.

8. Fouque, J.P. and Poliannikov, O., 2006, Time reversal detection in one-dimensional random media. Inverse Problems, 22(3), 903-922.

9. J. Garnier and K. Sølna, Cross correlation and deconvolution of noise signals in randomly layered media SIAM J. Imaging Sci., 3, 809-834, 2010.

10. Poliannikov, O. and Fouque, J.P., 2005, Detection of a reflective layer in a random medium using time reversal. In: Proceedings of the 2005 Int. Conf. on Acoustics, Speech and Signal Procesing, March.

11. Burridge, R., Papanicolaou, G., Sheng, P. and White, B., 1989, Probing a random medium with a pulse. SIAM J. Appl. Math., 49, 582-607.

12. Asch, M., Kohler, W., Papanicolaou, G., Postel, M. and White, B., 1991, Frequency content of randomly scattered signals. SIAM Review, 33, 519-626.

13. Alfaro Vigo, D., 2004, Time-reversed acoustics in a randomly changing medium. PhD thesis, IMPA, Rio de Janeiro, Brazil.

14. Alfaro Vigo, D., Fouque, J.P., Garnier, J. and Nachbin, A., 2004, Robustness of time reversal for waves in time-dependent random media. Stoch. Processes Appl., 113(2), 289-313.

15. Krylov, N. and Rozovskii, B., 1982, Stochastic partial differential equations and diffusion processes. Uspekhi Mat. Nauk, 37(6), 75-95 (transl. in Russian Math. Surveys, 37:6(1982)).

16. Kunita, H., 1990 Stochastic flows and stochastic differential equations, Cambridge Studies in Advanced Mathematics Vol. 24 (Cambridge: Cambridge University Press).

17. Asch, M., Papanicolaou, G., Postel, M., Sheng, P. and White, B., 1990, Frequency content of randomly scattered signals. Part I. Wave Motion, 12, 429-450.

18. Papanicolaou, G., Postel, M., Sheng, P. and White, B., 1990, Frequency content of randomly scattered signals. Part II: Inversion. Wave Motion, 12, 527-549.

19. Rozanov, Y.A., 1967, Stationary random processes (Holden-Day) (translated from russian by A. Feinstein).

20. Serfling, R., 1980, Approximation theorems of mathematical statistics (New York: John Wiley \& Sons).

21. Lehmann, E., 1986 Testing statistical hypothesis, second (New York: John Wiley \& Sons).

22. Johnson, N. and Kotz, S., 1970, Distributions in statistics-Continuous Univariate Distributions, Vol. 2 (New York: John Wiley \& Sons).

23. Ma, Y., Lim, T. and Pasupathy, S., 2002, Error probability for coherent and differential PSK over arbitrary Rician fading channels with multiple cochannel interferers. IEEE Trans. Commun., 50(3), 429-441.

24. Böttcher, A. and Silbermann, B., 1998, Introduction to large truncated Toeplitz matrices (Springer-Verlag).

25. Alfaro Vigo, D., Correia, A.S. and Nachbin, A., 2007, Complete time-reversed refocusing in reflection with an acoustic Lagrangian model. Commun. Math. Sci., 5(1), 161-185. 
Departamento de Ciência da Computação, Instituto de Matemática, Universidade Federal do Rio de Janeiro, Caixa Postal: 68530, Rio de Janeiro, 21945-970, Brazil

E-mail address: dgalfaro@dcc.ufrj.br

Dept. of Mathematics, University of California at Irvine, Irvine, CA 92697-3875, USA

E-mail address: ksolna@math.uci.edu 